\title{
Asymptotically linear systems near and at resonance
}

\author{
Maya Chhetri ${ }^{1}$ and Petr Girg ${ }^{2,3^{*}}$
}

\author{
"Correspondence: \\ pgirg@kma.zcu.cz \\ ${ }^{2}$ NTIS - New Technologies for the \\ Information Society, European \\ Centre of Excellence, Faculty of \\ Applied Sciences, University of West \\ Bohemia, Univerzitní 8, Plzeň, 30614, \\ Czech Republic \\ ${ }^{3} \mathrm{KMA}-\mathrm{FAV}$, Západočeská Univerzita \\ v Plzni, Univerzitní 22, Plzeň, 30614, \\ Czech Republic \\ Full list of author information is \\ available at the end of the article
}

\begin{abstract}
This paper deals with an elliptic system of the form $-\Delta u=\lambda \theta_{1} a(x) v+f(\lambda, x, v)$ in $\Omega$, $-\Delta v=\lambda \theta_{2} a(x) u+g(\lambda, x, u)$ in $\Omega, u=0=v$ on $\partial \Omega$, where $\lambda \in \mathbb{R}$ is a parameter and $\Omega \subset \mathbb{R}^{N}(N \geq 1)$ is a bounded domain with $C^{2, \xi}$-boundary $\partial \Omega, \xi \in(0,1)$ (a bounded open interval if $N=1)$. Here $a(x) \in L^{\infty}(\Omega)$ with $a(x)>0$ a.e. in $\Omega$ and $\theta_{1}, \theta_{2}>0$ are constants. The nonlinear perturbations $f, g: \mathbb{R} \times \Omega \times \mathbb{R} \rightarrow \mathbb{R}$ are Carathéodory functions that are sublinear at infinity. We provide sufficient conditions for determining the $\boldsymbol{\lambda}$-direction to which a continuum of positive and negative solutions emanates from infinity at the first eigenvalue of the associated linear problem. Furthermore, as a consequence of main results, we also provide sufficient condition for the solvability of a class of asymptotically linear system near and at resonance satisfying Landesman-Lazer type conditions.

MSC: $35 J 60$
\end{abstract}

Keywords: elliptic systems; asymptotically linear; bifurcation from infinity; semipositone; resonance; Landesman-Lazer condition

\section{Introduction}

We consider an elliptic system of the form

$$
\left.\begin{array}{ll}
-\Delta u=\lambda \theta_{1} a(x) v+f(\lambda, x, v) & \text { in } \Omega, \\
-\Delta v=\lambda \theta_{2} a(x) u+g(\lambda, x, u) & \text { in } \Omega, \\
u=0=v \quad \text { on } \partial \Omega,
\end{array}\right\}
$$

where $\Omega \subset \mathbb{R}^{N}(N \geq 1)$ is a bounded domain with $C^{2, \xi}$-boundary $\partial \Omega, \xi \in(0,1)$ (a bounded open interval if $N=1$ ). We will assume that $\lambda \in \mathbb{R}$ is a parameter, $\theta_{1}, \theta_{2}>0$ are constants, $a(x)>0$ a.e. in $\Omega$ is an $L^{\infty}(\Omega)$ function. The nonlinear perturbations $f, g$ : $\mathbb{R} \times \Omega \times \mathbb{R} \rightarrow \mathbb{R}$ satisfy the following assumptions uniformly on compact intervals of $\lambda$ :

(H1) $f$ and $g$ are Carathéodory functions;

(H2) there exist $h \in L^{r}(\Omega), r>N$ with $h(x) \geq 0$ for a.e. $x \in \Omega$, and a constant $b \geq 0$ such that

$$
|f(\lambda, x, s)|,|g(\lambda, x, s)| \leq h(x)+b|s| \quad \text { for all } s \in \mathbb{R}
$$

\section{Springer}

(c) 2014 Chhetri and Girg; licensee Springer. This is an Open Access article distributed under the terms of the Creative Commons Attribution License (http://creativecommons.org/licenses/by/4.0), which permits unrestricted use, distribution, and reproduction in any medium, provided the original work is properly credited. 
(H3) $f$ and $g$ are sublinear at infinity, that is, for a.e. $x \in \Omega$

$$
\lim _{|s| \rightarrow \infty} \frac{f(\lambda, x, s)}{s}=0 \quad \text { and } \quad \lim _{|s| \rightarrow \infty} \frac{g(\lambda, x, s)}{s}=0 .
$$

Therefore, the system (1.1) is asymptotically linear at infinity.

In this paper, we are interested in studying positive solutions of (1.1) via bifurcation theory. For this, we define the underlying space

$$
E:=\left(W_{0}^{1,2}(\Omega) \cap W^{2, r}(\Omega)\right) \times\left(W_{0}^{1,2}(\Omega) \cap W^{2, r}(\Omega)\right)
$$

for $r>N$, endowed with the norm $\left\|\left(w_{1}, w_{2}\right)\right\|_{E}:=\left\|w_{1}\right\|_{W^{2, r}(\Omega)}+\left\|w_{2}\right\|_{W^{2, r}(\Omega)}$. We say that $(\lambda,(u, v)) \in \mathbb{R} \times E$ is a solution of (1.1) if $(\lambda,(u, v))$ solves (1.1) in the strong sense, that is, $u, v \in W^{2, r}(\Omega)$ and $(\lambda,(u, v))$ satisfies (1.1) almost everywhere in $\Omega$. Further, if $u>0(<0)$ and $v>0(<0)$ almost everywhere in $\Omega$ then we say that $(\lambda,(u, v))$ is a positive (negative) solution of (1.1).

We say that $\lambda_{\infty} \in \mathbb{R}$ is a bifurcation point from infinity if the solution set

$$
\mathscr{T}:=\{(\lambda,(u, v)) \in \mathbb{R} \times E:(\lambda,(u, v)) \text { solves }(1.1)\}
$$

contains a sequence $\left\{\left(\lambda_{n},\left(u_{n}, v_{n}\right)\right)\right\}$ such that

$$
\lambda_{n} \rightarrow \lambda_{\infty} \quad \text { and } \quad\left\|\left(u_{n}, v_{n}\right)\right\|_{E} \rightarrow \infty
$$

By a continuum of solutions of (1.1) we mean $\mathfrak{C} \subset \mathscr{T}$ which is closed and connected.

A continuum $\mathfrak{C}$ bifurcates from infinity at $\lambda_{\infty} \in \mathbb{R}$ if there exists a sequence of solutions $\left\{\left(\lambda_{n},\left(u_{n}, v_{n}\right)\right)\right\}_{n=1}^{\infty} \subset \mathfrak{C}$ such that $\lambda_{n} \rightarrow \lambda_{\infty}$ and $\left\|\left(u_{n}, v_{n}\right)\right\|_{E} \rightarrow+\infty$ as $n \rightarrow \infty$.

Let $\mu_{1}$ be the principal eigenvalue of

$$
\left.\begin{array}{ll}
-\Delta \phi=\lambda a(x) \phi & \text { in } \Omega, \\
\phi=0 & \text { on } \partial \Omega,
\end{array}\right\}
$$

and $\phi_{1}$ be the corresponding eigenfunction. Without loss of generality, we normalize the eigenfunction such that $\phi_{1}>0$ in $\Omega$ and

$$
2 \sqrt{\theta_{1} \theta_{2}} \int_{\Omega} a(x) \phi_{1}^{2} \mathrm{~d} x=1 .
$$

Define $v_{1}:=\frac{\mu_{1}}{\sqrt{\theta_{1} \theta_{2}}}$. We will show, in Appendix 2, that $v_{1}$ is a simple eigenvalue of the linear operator associated with the linear part of (1.1), that is, of

$$
\left.\begin{array}{l}
-\Delta w_{1}=\lambda \theta_{1} a(x) w_{2} \quad \text { in } \Omega, \\
-\Delta w_{2}=\lambda \theta_{2} a(x) w_{1} \quad \text { in } \Omega, \\
w_{1}=0=w_{2} \quad \text { on } \partial \Omega .
\end{array}\right\}
$$

It is the only eigenvalue such that both components of its eigenfunction, $\left(\sqrt{\theta_{1}} \phi_{1}, \sqrt{\theta_{2}} \phi_{1}\right)$, are positive (or negative) in $\Omega$. Further, for each $|k| \in \mathbb{N}, v_{k}$ denotes the eigenvalue of (1.4). For a detailed discussion of the eigenvalue problem (1.4), see Appendix 2.

Now we state our main results. 
Theorem 1.1 Let (H1)-(H3) hold. Then there exist $\varepsilon, K>0$ such that any solution $(\lambda,(u, v)) \in \mathscr{T}$ with $v_{1}-\varepsilon \leq \lambda \leq v_{1}+\varepsilon$ and $\|(u, v)\|_{E}>K$ satisfy $u>0$ and $v>0$ or $u<0$ and $v<0$, and $v_{k} \notin\left[v_{1}+\varepsilon, v_{1}-\varepsilon\right]$ for any $|k| \in \mathbb{N}$ with $k \neq 1$. Moreover, there are continua $\mathscr{D}_{\nu_{1}}^{+}, \mathscr{D}_{v_{1}}^{-} \subset \mathscr{T}$ bifurcating from infinity at $v_{1}$ containing positive and negative solutions, respectively.

Theorem 1.1 establishes the existence of positive and negative solutions of (1.1) near $v_{1}$. In the next two theorems we determine the $\lambda$-direction to which the continua from Theorem 1.1 bifurcate. For this we impose additional conditions on the perturbations $f$ and $g$ below.

For $\alpha \geq 0$, define

$$
\underline{A}_{\alpha}^{ \pm}:=\liminf _{(\lambda, s) \rightarrow\left(\nu_{1}, \pm \infty\right)}\left[\sqrt{\theta_{2}} f(\lambda, x, s)+\sqrt{\theta_{1}} g(\lambda, x, s)\right] s^{\alpha}
$$

and

$$
\bar{A}_{\alpha}^{ \pm}:=\limsup _{(\lambda, s) \rightarrow\left(\nu_{1}, \pm \infty\right)}\left[\sqrt{\theta_{2}} f(\lambda, x, s)+\sqrt{\theta_{1}} g(\lambda, x, s)\right] s^{\alpha} .
$$

Suppose there exist $0 \leq \alpha_{i}<2-\frac{1}{r}$ and nonnegative functions $B_{i} \in L^{r}(\Omega)$ for $i=1, \ldots, 4$ such that

$$
\begin{array}{ll}
(\mathrm{H} 4+) & f(\lambda, x, s)(1+s)^{\alpha_{1}}, g(\lambda, x, s)(1+s)^{\alpha_{1}} \geq-B_{1}(x) \quad \forall s \geq 0, \\
(\mathrm{H} 4-) \quad-f(\lambda, x, s)|s-1|^{\alpha_{2}},-g(\lambda, x, s)|s-1|^{\alpha_{2}} \geq-B_{2}(x) \quad \forall s \leq 0, \\
(\mathrm{H} 5+) \quad f(\lambda, x, s)(1+s)^{\alpha_{3}}, g(\lambda, x, s)(1+s)^{\alpha_{3}} \leq B_{3}(x) \quad \forall s \geq 0, \\
(\mathrm{H} 5-) \quad-f(\lambda, x, s)|s-1|^{\alpha_{4}},-g(\lambda, x, s)|s-1|^{\alpha_{4}} \leq B_{4}(x) \quad \forall s \leq 0 .
\end{array}
$$

Now let $\varepsilon, K, \mathscr{D}_{v_{1}}^{+}, \mathscr{D}_{\nu_{1}}^{-}$be as in Theorem 1.1. Then we prove the following results.

Theorem 1.2 Suppose the hypotheses of Theorem 1.1 hold. If $(\mathrm{H} 4+)((\mathrm{H} 4-))$ holds and

$$
\int_{\Omega} \underline{A}_{\alpha_{1}}^{+}(x) \phi_{1}^{1-\alpha_{1}}(x) \mathrm{d} x>0\left(\int_{\Omega} \bar{A}_{\alpha_{2}}(x) \phi_{1}^{1-\alpha_{2}}(x) \mathrm{d} x<0\right)
$$

then

(I) there is no positive (negative) solution $(\lambda,(u, v)) \in \mathscr{T}$ satisfying $v_{1} \leq \lambda \leq v_{1}+\varepsilon$ and $\|(u, v)\|_{E}>K$, and

(II) the continuum $\mathscr{D}_{v_{1}}^{+}\left(\mathscr{D}_{v_{1}}^{-}\right) \subset \mathscr{T}$, consisting of positive (negative) solutions, bifurcates from infinity at $v_{1}$ to the left.

Theorem 1.3 Suppose the hypotheses of Theorem 1.1 hold. If (H5+) ((H5-)) holds and

$$
\int_{\Omega} \bar{A}_{\alpha_{3}}^{+}(x) \phi_{1}^{1-\alpha_{3}}(x) \mathrm{d} x<0\left(\int_{\Omega} \underline{A}_{\alpha_{4}}^{-}(x) \phi_{1}^{1-\alpha_{4}}(x) \mathrm{d} x>0\right),
$$


(I) there is no positive (negative) solution $(\lambda,(u, v)) \in \mathscr{T}$ satisfying $v_{1}-\varepsilon \leq \lambda \leq \nu_{1}$ and $\|(u, v)\|_{E}>K$, and

(II) the continuum $\mathscr{D}_{\nu_{1}}^{+}\left(\mathscr{D}_{\nu_{1}}^{-}\right) \subset \mathscr{T}$, consisting of positive (negative) solutions, bifurcates from infinity at $v_{1}$ to the right.

This paper is motivated by the results obtained for the scalar case in [1, Theorem 3 and Theorem 4] and in [2, Theorem 1]. The goal of the present paper is to extend the above results to systems for positive and negative solutions. We prove our results by heavily utilizing a version of Lyapunov-Schmidt reduction method applied to a bifurcation from infinity.

We do not require any sign conditions on the nonlinear perturbations $f$ and $g$ near the origin. Thus Theorem 1.2 and Theorem 1.3 apply to both positone and semipositone type right-hand sides of (1.1) for positive solutions. See [3] and [4], where an asymptotically linear system is considered but nonlinearities are singular at the origin. The existence of a positive solution to the left of $v_{1}$ is established in [3] using Schauder fixed point theory and to the left of $v_{1}$ but away from $v_{1}$ is established in [4] using the sub and supersolution methods. In $[5,6]$, the authors consider a more general system, which includes system such as (1.1) as a special case. Their result, with additional assumptions on nonlinearities, shows that (1.1) has a positive solution in the right neighborhood of $v_{1}$ using critical point theory. These papers provide the existence of solutions, but do not provide information on the connectivity of the solution set. Therefore Theorem 1.2 and Theorem 1.3 complement these existence results.

As a by-product of the theorems above, we have the following existence results for (1.1) at and near resonance.

Theorem 1.4 Suppose the hypotheses of Theorem 1.1 hold. If $(\mathrm{H} 4+)$ and (H4-) hold, and

$$
\int_{\Omega} \bar{A}_{\alpha_{2}}^{-}(x) \phi_{1}^{1-\alpha_{2}}(x) \mathrm{d} x<0<\int_{\Omega} \underline{A}_{\alpha_{1}}^{+}(x) \phi_{1}^{1-\alpha_{1}}(x) \mathrm{d} x,
$$

then (1.1) has

(a) at least two solutions (one positive and one negative) for $\lambda \in\left[v_{1}-\varepsilon, v_{1}\right)$, and

(b) at least one solution for $\lambda \in\left[v_{1}, v_{1}+\varepsilon\right]$.

Theorem 1.5 Suppose the hypotheses of Theorem 1.1 hold. If (H5+) and (H5-) hold, and

$$
\int_{\Omega} \bar{A}_{\alpha_{3}}^{+}(x) \phi_{1}^{1-\alpha_{3}}(x) \mathrm{d} x<0<\int_{\Omega} \underline{A}_{\alpha_{4}}^{-}(x) \phi_{1}^{1-\alpha_{4}}(x) \mathrm{d} x
$$

then (1.1) has

(a) at least one solution for $\lambda \in\left[v_{1}-\varepsilon, v_{1}\right]$, and

(b) at least two solutions (one positive and one negative) for $\lambda \in\left(v_{1}, v_{1}+\varepsilon\right]$.

Theorem 1.4 and Theorem 1.5 generalize results for the scalar case in [1, Theorem 19] and [7, Theorem 9] to systems at the principal eigenvalue $v_{1}$. Our results also complement [8, Theorem 2.1]. Also see [9-11] and [12], where existence results were discussed at and/or near resonance. We prove our results as a direct consequence of our main theorems. More precisely, Theorem 1.2 implies Theorem 1.4 and Theorem 1.3 implies Theorem 1.5. 
Finally, we consider a straightforward extension of Landesman-Lazer type conditions initiated in the celebrated paper [13] by Landesman and Lazer to a system. In particular, we provide the solvability of the following system at resonance,

$$
\begin{aligned}
& -\Delta u=v_{1} \theta_{1} a(x) v+\tilde{f}(v)+h_{1}(x) \quad \text { in } \Omega, \\
& -\Delta v=v_{1} \theta_{2} a(x) u+\tilde{g}(u)+h_{2}(x) \text { in } \Omega \text {, } \\
& u=0=v \quad \text { on } \partial \Omega \text {. }
\end{aligned}
$$

We assume $a(x), \theta_{1}, \theta_{2}$ as before. Let $\tilde{f}, \tilde{g}: \mathbb{R} \rightarrow \mathbb{R}$ be continuous functions and $h_{i} \in L^{r}(\Omega)$ with $r>N$ satisfying

$$
\tilde{f}_{ \pm \infty}=\lim _{s \rightarrow \pm \infty} \tilde{f}(s) \in \mathbb{R}, \quad \tilde{g}_{ \pm \infty}=\lim _{s \rightarrow \pm \infty} \tilde{g}(s) \in \mathbb{R}
$$

Then we prove the following existence results, as corollaries of Theorem 1.4 and Theorem 1.5 , respectively.

\section{Corollary 1.6 Suppose}

$$
\begin{aligned}
& \int_{\Omega}\left(\sqrt{\theta_{2}} \tilde{f}_{-\infty}+\sqrt{\theta_{1}} \tilde{g}_{-\infty}\right) \phi_{1} \mathrm{~d} x \\
& \quad<-\int_{\Omega}\left(\sqrt{\theta_{2}} h_{1}+\sqrt{\theta_{1}} h_{2}\right) \phi_{1} \mathrm{~d} x<\int_{\Omega}\left(\sqrt{\theta_{2}} \tilde{f}_{+\infty}+\sqrt{\theta_{1}} \tilde{g}_{+\infty}\right) \phi_{1} \mathrm{~d} x .
\end{aligned}
$$

Then (1.9) has a solution.

\section{Corollary 1.7 Suppose}

$$
\begin{aligned}
& \int_{\Omega}\left(\sqrt{\theta_{2}} \tilde{f}_{-\infty}+\sqrt{\theta_{1}} \tilde{g}_{-\infty}\right) \phi_{1} \mathrm{~d} x \\
& \quad>-\int_{\Omega}\left(\sqrt{\theta_{2}} h_{1}+\sqrt{\theta_{1}} h_{2}\right) \phi_{1} \mathrm{~d} x>\int_{\Omega}\left(\sqrt{\theta_{2}} \tilde{f}_{+\infty}+\sqrt{\theta_{1}} \tilde{g}_{+\infty}\right) \phi_{1} \mathrm{~d} x .
\end{aligned}
$$

Then (1.9) has a solution.

Similar results were obtained in [10, Theorem 5.1]. The author uses continuation of solution with respect to a parameter using the implicit function theorem and hence requires the autonomous part of the nonlinear perturbations to be $C^{2}$ smooth.

Remark 1.8 In Figure 1, any solution in both dark and light gray regions will either be positive or negative by Theorem 1.1. There is no positive or negative solution in the dark gray regions due to Theorem 1.2. Solutions in the light gray region on $\mathscr{D}_{\nu_{1}}^{+}$are positive and on $\mathscr{D}_{v_{1}}^{-}$are negative. Dashed points are a sequence of solutions approaching the solution of the resonant problem at $v_{1}$ from the right (see proof of Theorem 1.4). The vertical axis is given by

$$
t^{-1}=\int_{\Omega} a(x)(u, v) \cdot\left(\sqrt{\theta_{2}} \phi_{1}, \sqrt{\theta_{1}} \phi_{1}\right) \mathrm{d} x
$$




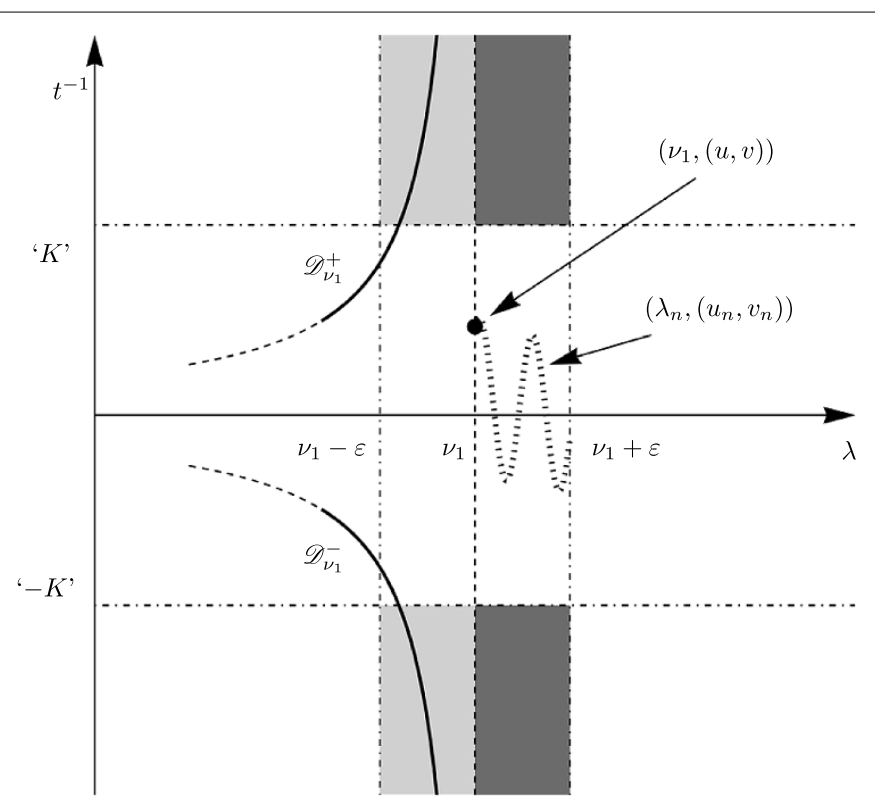

Figure 1 Results from Theorems 1.1-1.2 and Theorem 1.4. See Remark 1.8 for details.

in order to distinguish positive and negative solutions in the neighborhood of $v_{1}$. Here the symbol '. ' denotes the usual scalar product in $\mathbb{R}^{2}$. To understand the diagram it is helpful to observe that $\left|t^{-1}\right| \approx\|(u, v)\|_{E}$ for solutions with $\lambda$ close to $v_{1}$. See (4.4) for a precise statement. Values of $t^{-1}$ corresponding to the value $K$ from Theorem 1.1 are denoted by ' $K$ ' and ' $-K$ '.

In Section 2, we set up the functional framework for our problem to apply the abstract bifurcation theory discussed in Appendix 1. In Section 3, a variant of Krasnosel'skii's necessary condition for a bifurcation from infinity is discussed. In Section 4, we prove Theorems 1.1-1.3. In Section 5, we prove Theorems 1.4-1.5, and Corollaries 1.6-1.7. In Section 6, we provide several examples of $f$ and $g$ that satisfy the hypotheses of Theorems 1.1-1.3 and Corollaries 1.6-1.7. In Appendix 1, we discuss the abstract bifurcation theory (Rabinowitz and Dancer type) which we use in our analysis. In Appendix 2, we study the spectral properties of the eigenvalue problem (1.4). In Appendix 3, we provide the proof of (2.2) claimed in Section 2.

\section{Functional framework}

In this section, we set up functional framework that enables us to treat our problem using Proposition A.1 discussed in Appendix 1.

Due to the growth condition (H2) imposed on $f$ and $g$ and the fact that $a \in L^{\infty}(\Omega)$, all solutions $(u, v)$ of any elliptic system throughout this paper are understood in the strong sense, which means $(u, v) \in E$ (bootstrap method and [14, Theorem 9.15]) and they satisfy the corresponding pde a.e. in $\Omega$. Since, for any $r>N, W^{2, r}(\Omega) \hookrightarrow C^{1, \eta}(\bar{\Omega})$ for some $\eta \in$ $(0,1)$, the boundary conditions are satisfied in the usual sense.

The abstract setting of our problem is

$$
(u, v)=\lambda L(u, v)+H(\lambda,(u, v)),
$$


where $L: E \rightarrow E$ denotes the mapping

$$
(u, v) \mapsto(-\Delta)^{-1} a(x)\left(\theta_{1} v, \theta_{2} u\right)
$$

and $H: \mathbb{R} \times E \rightarrow E$ denotes the mapping

$$
(\lambda,(u, v)) \mapsto(-\Delta)^{-1}(f(\lambda, x, v), g(\lambda, x, u)) .
$$

The solution operator $(-\Delta)^{-1}: L^{r}(\Omega) \rightarrow W_{0}^{1,2}(\Omega) \cap W^{2, r}(\Omega), h \mapsto w$, associated to the problem

$$
-\Delta w=h \quad \text { in } \Omega, \quad w=0 \quad \text { on } \partial \Omega
$$

is well defined, obviously linear, continuous, and compact. Thus $L$ is linear, continuous, and compact. Since $f$ and $g$ are Carathéodory functions satisfying (H2), the corresponding Nemytski operator, denoted again by $f$ and $g$, maps $W^{2, r}(\Omega) \rightarrow L^{r}(\Omega)$ continuously (see [15, Theorem 2.3]). Thus by the compactness of $(-\Delta)^{-1}$, we find that $H(\lambda,(u, v))$ is continuous and compact.

Claim: $H$ satisfies

$$
\lim _{\|(u, v)\|_{E} \rightarrow+\infty} \frac{\|H(\lambda, u, v)\|_{E}}{\|(u, v)\|_{E}}=0
$$

uniformly for $\lambda$ in compact intervals. See Appendix 3 for a proof.

In order to satisfy the assumptions of Proposition A.1, we use the Kelvin transform

$$
\left(w_{1}, w_{2}\right):=\frac{(u, v)}{\|(u, v)\|_{E}^{2}}
$$

and define $\mathcal{N}: \mathbb{R} \times E \rightarrow L^{r}(\Omega) \times L^{r}(\Omega)$ by

$$
\mathcal{N}\left(\lambda,\left(w_{1}, w_{2}\right)\right):= \begin{cases}\left\|\left(w_{1}, w_{2}\right)\right\|_{E}^{2}\left(f\left(\lambda, x, \frac{w_{2}}{\left\|\left(w_{1}, w_{2}\right)\right\|_{E}^{2}}\right), g\left(\lambda, x, \frac{w_{1}}{\left\|\left(w_{1}, w_{2}\right)\right\|_{E}^{2}}\right)\right) ; & \left(w_{1}, w_{2}\right) \neq(0,0), \\ 0 ; & \left(w_{1}, w_{2}\right)=(0,0) .\end{cases}
$$

Clearly $\mathcal{N}$ is continuous for $\left(w_{1}, w_{2}\right) \neq(0,0)$ on compact intervals of $\lambda$. The continuity at $\left(w_{1}, w_{2}\right)=(0,0)$ follows from the fact that

$$
\int_{\Omega}\left|f\left(\lambda, x, \frac{w_{2}}{\left\|\left(w_{1}, w_{2}\right)\right\|_{E}^{2}}\right)\left\|\left(w_{1}, w_{2}\right)\right\|_{E}^{2}\right|^{r} \mathrm{~d} x=\int_{\Omega}\left|\frac{f(\lambda, x, v)}{\|(u, v)\|_{E}^{2}}\right|^{r} \mathrm{~d} x \rightarrow 0
$$

as $\|(u, v)\|_{E} \rightarrow \infty$ and hence as $\left\|\left(w_{1}, w_{2}\right)\right\|_{E} \rightarrow 0$. Similarly for $g$. Due to the compactness of $(-\Delta)^{-1}: L^{r}(\Omega) \rightarrow W^{2, r}(\Omega) \cap W_{0}^{1,2}(\Omega)$ it follows that ${ }_{0} H: \mathbb{R} \times E \rightarrow E$ defined as

$$
{ }_{0} H\left(\lambda,\left(w_{1}, w_{2}\right)\right):= \begin{cases}\left\|\left(w_{1}, w_{2}\right)\right\|_{E}^{2} H\left(\lambda,\left(w_{1}, w_{2}\right) /\left\|\left(w_{1}, w_{2}\right)\right\|_{E}^{2}\right) ; & \left(w_{1}, w_{2}\right) \neq(0,0), \\ 0 ; & \left(w_{1}, w_{2}\right)=(0,0),\end{cases}
$$

is continuous and compact. Therefore the operators $L, H$, and ${ }_{0} H$ satisfy the hypotheses of Proposition A.1. 


\section{Bifurcation from eigenvalue}

The following proposition is a variant of necessary condition for a bifurcation from infinity due to Krasnosel'skii [16, Statement, p.194]. This variant provides extra information on the $C^{1, \eta}(\bar{\Omega})$ convergence which is crucial in determining the definite sign (positive or negative) of solutions bifurcating from infinity.

Proposition 3.1 If $v^{*}$ is a bifurcation point from infinity for (1.1), then $v^{*}=v_{l}$ for some $l:|l| \in \mathbb{N}$. Moreover, for any sequence $\left(\lambda_{n},\left(u_{n}, v_{n}\right)\right) \in \mathbb{R} \times E$ with $\lambda_{n} \rightarrow v^{*}$ and $\left\|\left(u_{n}, v_{n}\right)\right\|_{E} \rightarrow$ $+\infty$ as $n \rightarrow+\infty$, there exists an eigenfunction $\left(w_{1}^{*}, w_{2}^{*}\right) \in$ E satisfying $\left\|\left(w_{1}^{*}, w_{2}^{*}\right)\right\|_{E}=1$ corresponding to the eigenvalue $v^{*}$, and a subsequence $\left(\lambda_{n_{k}},\left(u_{n_{k}}, v_{n_{k}}\right)\right)$ of $\left(\lambda_{n},\left(u_{n}, v_{n}\right)\right)$ such that

$$
\lim _{n_{k} \rightarrow+\infty} \frac{\left(u_{n_{k}}, v_{n_{k}}\right)}{\left\|\left(u_{n_{k}}, v_{n_{k}}\right)\right\|_{E}}=\left(w_{1}^{*}, w_{2}^{*}\right)
$$

where the convergence is in $C^{1, \eta}(\bar{\Omega}) \times C^{1, \eta}(\bar{\Omega})$, for some $\eta \in(0,1)$.

In particular, for $v^{*}=v_{1}$, any sequence $\left(\lambda_{n},\left(u_{n}, v_{n}\right)\right) \in \mathbb{R} \times E$ with $\lambda_{n} \rightarrow v_{1}$ and $\|\left(u_{n}\right.$, $\left.v_{n}\right) \|_{E} \rightarrow+\infty$ as $n \rightarrow+\infty$ can be split into two subsequences one of which satisfies

$$
\lim _{n_{k} \rightarrow+\infty} \frac{\left(u_{n_{k}}, v_{n_{k}}\right)}{\left\|\left(u_{n_{k}}, v_{n_{k}}\right)\right\|_{E}}= \pm \frac{\left(\sqrt{\theta_{1}} \phi_{1}, \sqrt{\theta_{2}} \phi_{1}\right)}{\left\|\left(\sqrt{\theta_{1}} \phi_{1}, \sqrt{\theta_{2}} \phi_{1}\right)\right\|_{E}}
$$

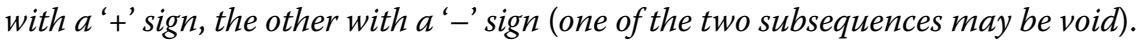

Proof Let $\left(\lambda_{n},\left(u_{n}, v_{n}\right)\right) \in \mathbb{R} \times E$ be solutions of (1.1) such that $\left\|\left(u_{n}, v_{n}\right)\right\|_{E} \rightarrow+\infty$ and $\lambda_{n} \in$ $\Lambda$, where $\Lambda \subset \mathbb{R}$ is a compact interval. Then $\left(w_{1, n}, w_{2, n}\right)=\frac{\left(u_{n}, v_{n}\right)}{\left\|\left(u_{n}, v_{n}\right)\right\|_{E}}$ satisfies

$$
\begin{aligned}
& w_{1, n}=(-\Delta)^{-1}\left(\lambda_{n} \theta_{1} a(x) w_{2, n}+\frac{f\left(\lambda_{n}, x, v_{n}\right)}{\left\|\left(u_{n}, v_{n}\right)\right\|_{E}}\right), \\
& w_{2, n}=(-\Delta)^{-1}\left(\lambda_{n} \theta_{2} a(x) w_{1, n}+\frac{g\left(\lambda_{n}, x, u_{n}\right)}{\left\|\left(u_{n}, v_{n}\right)\right\|_{E}}\right) .
\end{aligned}
$$

Owing to (C.1), we find that the terms on the right-hand sides are bounded in $L^{r}(\Omega)$ (independent of $n$ ). Hence $\left\|w_{1, n}\right\|_{W^{2, r}(\Omega)}$ and $\left\|w_{2, n}\right\|_{W^{2, r}(\Omega)}$ are bounded (independent of $n$ ) and so are $\left\|w_{1, n}\right\|_{C^{1, \eta}(\bar{\Omega})}$ and $\left\|w_{2, n}\right\|_{C^{1, \eta}(\bar{\Omega})}$, for some $\eta \in(0,1)$. Since $C^{1, \eta^{\prime}}(\bar{\Omega}) \hookrightarrow C^{1, \eta}(\bar{\Omega})$ compactly for $\eta^{\prime} \in(0, \eta)$, passing to a subsequence, $w_{1, n} \rightarrow w_{1}^{*}, w_{2, n} \rightarrow w_{2}^{*}$ in $C^{1, \eta^{\prime}}(\bar{\Omega})$ and $\lambda_{n} \rightarrow \lambda^{*} \in \Lambda$. Therefore $\left(\lambda^{*},\left(w_{1}^{*}, w_{2}^{*}\right)\right)$ satisfies

$$
\begin{aligned}
& w_{1}^{*}=(-\Delta)^{-1} \lambda^{*} \theta_{1} a(x) w_{2}^{*}, \\
& w_{2}^{*}=(-\Delta)^{-1} \lambda^{*} \theta_{2} a(x) w_{1}^{*},
\end{aligned}
$$

and hence satisfies (1.4). Since $\left\|\left(w_{1}^{*}, w_{2}^{*}\right)\right\|_{E}=1, \lambda^{*} \in \Lambda$ must be an eigenvalue of (1.4) and $\left(w_{1}^{*}, w_{2}^{*}\right) \in E$ a corresponding normalized eigenvector.

For the case $v^{*}=v_{1}$, the statement follows from the fact that $v_{1}$ is simple.

\section{Proof of main results}

Proof of Theorem 1.1 We will use the Lyapunov-Schmitt reduction method combined with a bifurcation from infinity. We split our underlying space $E=\operatorname{span}\left\{\left(\sqrt{\theta}_{1} \phi_{1}, \sqrt{\theta}_{2} \phi_{1}\right)\right\} \oplus E_{1}$, 
where

$$
E_{1}:=\left\{\left(z_{1}, z_{2}\right) \in E: \int_{\Omega} a(x)\left(z_{1}, z_{2}\right) \cdot\left(\sqrt{\theta}_{2} \phi_{1}, \sqrt{\theta}_{1} \phi_{1}\right) \mathrm{d} x=0\right\},
$$

and $\left(\sqrt{\theta}_{1} \phi_{1} \sqrt{\theta}_{2} \phi_{1}\right)$ and $\left(\sqrt{\theta}_{2} \phi_{1}, \sqrt{\theta}_{1} \phi_{1}\right)$ are the eigenfunctions of the linear operator $L$ and its adjoint $L^{*}$, respectively, corresponding to the eigenvalue $v_{1}$ (see Appendix 2 for details).

By Proposition 3.1, for large $n,\left(u_{n}, v_{n}\right)$ takes the form

$$
\left(u_{n}, v_{n}\right)=t_{n}^{-1}\left(\sqrt{\theta_{1}} \phi_{1}+u_{n}^{\top}, \sqrt{\theta_{2}} \phi_{1}+v_{n}^{\top}\right),
$$

where $t_{n} \rightarrow 0, t_{n} \neq 0$, and $\left(u_{n}^{\top}, v_{n}^{\top}\right) \in E_{1}$, that is, they satisfy the 'orthogonality' condition

$$
\int_{\Omega} a(x)\left(\sqrt{\theta_{2}} u_{n}^{\top}+\sqrt{\theta_{1}} v_{n}^{\top}\right) \phi_{1} \mathrm{~d} x=0
$$

with $u_{n}^{\top} \rightarrow 0, v_{n}^{\top} \rightarrow 0$ in $C^{1, \eta}(\bar{\Omega})$ as $n \rightarrow+\infty$. It follows from a straightforward calculation, using (1.3), that $t_{n}^{-1}$ is the projection of $\left(u_{n}, v_{n}\right)$ to the subspace spanned by $\left(\sqrt{\theta_{1}} \phi_{1}, \sqrt{\theta_{2}} \phi_{1}\right)$. Indeed,

$$
\begin{aligned}
& \int_{\Omega} a(x)\left(u_{n}, v_{n}\right) \cdot\left(\sqrt{\theta_{2}} \phi_{1}, \sqrt{\theta_{1}} \phi_{1}\right) \mathrm{d} x \\
& \quad=\int_{\Omega} a(x) t_{n}^{-1}\left(\sqrt{\theta_{1}} \phi_{1}+u_{n}^{\top}, \sqrt{\theta_{2}} \phi_{1}+v_{n}^{\top}\right) \cdot\left(\sqrt{\theta_{2}} \phi_{1}, \sqrt{\theta_{1}} \phi_{1}\right) \mathrm{d} x \\
& \quad=t_{n}^{-1} \in \mathbb{R} .
\end{aligned}
$$

It is important to observe that $\left|t_{n}\right|^{-1} \rightarrow \infty$ if and only if $\left\|\left(u_{n}, v_{n}\right)\right\|_{E} \rightarrow \infty$ as $n \rightarrow \infty$. Indeed, it follows from (4.3), (1.3) and (3.2) that

$$
\frac{\left|t_{n}\right|^{-1}}{\left\|\left(u_{n}, v_{n}\right)\right\|_{E}} \rightarrow \frac{1}{\left\|\left(\sqrt{\theta_{1}} \phi_{1}, \sqrt{\theta_{2}} \phi_{1}\right)\right\|_{E}} \quad \text { as } n \rightarrow \infty
$$

Therefore, since $\frac{\partial \phi_{1}}{\partial \vec{n}}<0$ on $\partial \Omega$, it follows from (4.1) that $u_{n}(x)>0(<0)$ and $v_{n}(x)>0$ $(<0)$ for $n$ sufficiently large. Thus there exist $\varepsilon, K>0$ such that any $\left(\lambda_{n},\left(u_{n}, v_{n}\right)\right) \in \mathscr{T}$ with $v_{1}-\varepsilon \leq \lambda_{n} \leq v_{1}+\varepsilon$ and $\left\|\left(u_{n}, v_{n}\right)\right\|_{E}>K$ satisfy $u_{n}>0(<0)$ and $v_{n}>0(<0)$ in $\Omega$ and $v_{k} \notin\left[v_{1}+\varepsilon, v_{1}-\varepsilon\right]$ for any $|k| \in \mathbb{N}$ with $k \neq 1$ for large $n$. This proves the first part of Theorem 1.1.

By Proposition A.1, there exist two continua $\mathscr{D}_{v_{1}}^{+} \subset \mathscr{T}$ and $\mathscr{D}_{v_{1}}^{-} \subset \mathscr{T}$ emanating, respectively, in the direction of positive and negative multiple of $\left(\sqrt{\theta_{1}} \phi_{1}, \sqrt{\theta}_{2} \phi_{1}\right)$ in $E$. Then in view of the $C^{1}$-regularity of solutions and Proposition 3.1, $\mathscr{D}_{v_{1}}^{+}\left(\mathscr{D}_{v_{1}}^{-}\right)$is the continuum containing large positive (negative) solutions corresponding to $t_{n}>0\left(t_{n}<0\right)$.

Proof of Theorem 1.2 We will first establish part (I) of Theorem 1.2 by determining the $\lambda$-direction of the bifurcation of positive solutions from infinity at $v_{1}$. Let $\left(\lambda_{n},\left(u_{n}, v_{n}\right)\right) \in \mathscr{T}$ be such that $\lambda_{n} \rightarrow v_{1}$ and $\left\|\left(u_{n}, v_{n}\right)\right\|_{E} \rightarrow+\infty$. Then by Proposition 3.1, $\left(u_{n}, v_{n}\right)$ takes the form

$$
\begin{aligned}
& u_{n}=t_{n}^{-1}\left(\sqrt{\theta_{1}} \phi_{1}+u_{n}^{\top}\right), \\
& v_{n}=t_{n}^{-1}\left(\sqrt{\theta_{2}} \phi_{1}+v_{n}^{\top}\right),
\end{aligned}
$$


where $t_{n}>0, t_{n} \rightarrow 0,\left\|u_{n}^{\top}\right\|_{C^{1, \eta}(\bar{\Omega})} \rightarrow 0$, and $\left\|v_{n}^{\top}\right\|_{C^{1, \eta}(\bar{\Omega})} \rightarrow 0$ as $n \rightarrow \infty$. Therefore $u_{n}>0$ and $v_{n}>0$ for large $n$.

Multiplying the first equation of (1.1) by the first component of $\left(\sqrt{\theta_{2}} \phi_{1}, \sqrt{\theta_{1}} \phi_{1}\right)$ (eigenfunction corresponding to the adjoint operator $L^{*}$ ), integrating over $\Omega$, and using (4.1), we obtain

$$
\begin{aligned}
& -\int_{\Omega} \Delta\left[t_{n}^{-1}\left(\sqrt{\theta_{1}} \phi_{1}+u_{n}^{\top}\right)\right] \sqrt{\theta_{2}} \phi_{1} \mathrm{~d} x \\
& \quad=\lambda_{n} \theta_{1} \sqrt{\theta_{2}} \int_{\Omega} a(x) t_{n}^{-1}\left(\sqrt{\theta_{2}} \phi_{1}+v_{n}^{\top}\right) \phi_{1} \mathrm{~d} x+\sqrt{\theta_{2}} \int_{\Omega} f\left(\lambda_{n}, x, v_{n}\right) \phi_{1} \mathrm{~d} x .
\end{aligned}
$$

This yields

$$
\begin{aligned}
\mu_{1} & \sqrt{\theta_{1} \theta_{2}} \int_{\Omega} a(x) \phi_{1}^{2} \mathrm{~d} x+\sqrt{\theta_{2}} \mu_{1} \int_{\Omega} a(x) u_{n}^{\top} \phi_{1} \mathrm{~d} x \\
= & \mu_{1} \sqrt{\theta_{1} \theta_{2}} \int_{\Omega} a(x) \phi_{1}^{2} \mathrm{~d} x+\left[\lambda_{n}-\frac{\mu_{1}}{\sqrt{\theta_{1} \theta_{2}}}\right] \theta_{1} \theta_{2} \int_{\Omega} a(x) \phi_{1}^{2} \mathrm{~d} x \\
& +\lambda_{n} \theta_{1} \sqrt{\theta_{2}} \int_{\Omega} a(x) v_{n}^{\top} \phi_{1} \mathrm{~d} x+t_{n} \sqrt{\theta_{2}} \int_{\Omega} f\left(\lambda_{n}, x, v_{n}\right) \phi_{1} \mathrm{~d} x,
\end{aligned}
$$

and, using (1.3), it simplifies to

$$
\begin{aligned}
\mu_{1} & \sqrt{\theta_{2}} \int_{\Omega} a(x) u_{n}^{\top} \phi_{1} \mathrm{~d} x \\
= & {\left[\lambda_{n}-\frac{\mu_{1}}{\sqrt{\theta_{1} \theta_{2}}}\right] \frac{\sqrt{\theta_{1} \theta_{2}}}{2} } \\
& +\lambda_{n} \theta_{1} \sqrt{\theta_{2}} \int_{\Omega} a(x) v_{n}^{\top} \phi_{1} \mathrm{~d} x+t_{n} \sqrt{\theta_{2}} \int_{\Omega} f\left(\lambda_{n}, x, v_{n}\right) \phi_{1} \mathrm{~d} x .
\end{aligned}
$$

Similarly, multiplying the second equation of (1.1) by the second component of $\left(\sqrt{\theta_{2}} \phi_{1}\right.$, $\left.\sqrt{\theta_{1}} \phi_{1}\right)$, and integrating over $\Omega$, we obtain

$$
\begin{aligned}
\mu_{1} & \sqrt{\theta_{1}} \int_{\Omega} a(x) v_{n}^{\top} \phi_{1} \mathrm{~d} x \\
= & {\left[\lambda_{n}-\frac{\mu_{1}}{\sqrt{\theta_{1} \theta_{2}}}\right] \frac{\sqrt{\theta_{1} \theta_{2}}}{2} } \\
& +\lambda_{n} \theta_{2} \sqrt{\theta_{1}} \int_{\Omega} a(x) u_{n}^{\top} \phi_{1} \mathrm{~d} x+t_{n} \sqrt{\theta_{1}} \int_{\Omega} g\left(\lambda_{n}, x, u_{n}\right) \phi_{1} \mathrm{~d} x .
\end{aligned}
$$

Adding (4.6) and (4.7), we have

$$
\begin{aligned}
& \mu_{1} \int_{\Omega} a(x)\left(\sqrt{\theta_{2}} u_{n}^{\top}+\sqrt{\theta_{1}} v_{n}^{\top}\right) \phi_{1} \mathrm{~d} x \\
& =\left[\lambda_{n}-\frac{\mu_{1}}{\sqrt{\theta_{1} \theta_{2}}}\right] \sqrt{\theta_{1} \theta_{2}}+\lambda_{n} \sqrt{\theta_{1} \theta_{2}} \int_{\Omega} a(x)\left(\sqrt{\theta_{2}} u_{n}^{\top}+\sqrt{\theta_{1}} v_{n}^{\top}\right) \phi_{1} \mathrm{~d} x \\
& \quad+t_{n} \int_{\Omega}\left(\sqrt{\theta_{2}} f\left(\lambda_{n}, x, v_{n}\right)+\sqrt{\theta_{1}} g\left(\lambda_{n}, x, u_{n}\right)\right) \phi_{1} \mathrm{~d} x .
\end{aligned}
$$


Using $\frac{\mu_{1}}{\sqrt{\theta_{1} \theta_{2}}}=v_{1}$ and (4.2), the above equation simplifies to

$$
\left[v_{1}-\lambda_{n}\right] \sqrt{\theta_{1} \theta_{2}}=t_{n} \int_{\Omega}\left(\sqrt{\theta_{2}} f\left(\lambda_{n}, x, v_{n}\right)+\sqrt{\theta_{1}} g\left(\lambda_{n}, x, u_{n}\right)\right) \phi_{1} \mathrm{~d} x .
$$

The previous equation gives rise to the following important identity for sufficiently large $n$ :

$$
\operatorname{sgn}\left[v_{1}-\lambda_{n}\right]=\operatorname{sgn}\left[\int_{\Omega}\left(\sqrt{\theta_{2}} f\left(\lambda_{n}, x, v_{n}\right)+\sqrt{\theta_{1}} g\left(\lambda_{n}, x, u_{n}\right)\right) \phi_{1} \mathrm{~d} x\right] .
$$

Proof of part (I) for positive solutions: Now we proceed to complete the proof of part (I) of Theorem 1.2 by contradiction. Suppose that $\lambda_{n} \in\left[v_{1}, v_{1}+\varepsilon\right]$ for all $n$ sufficiently large. This implies, by (4.8), that

$$
\left[\int_{\Omega}\left(\sqrt{\theta_{2}} f\left(\lambda_{n}, x, v_{n}\right)+\sqrt{\theta_{1}} g\left(\lambda_{n}, x, u_{n}\right)\right) \phi_{1} \mathrm{~d} x\right] \leq 0
$$

for $n$ sufficiently large.

On the other hand, since $u_{n}>0$ and $v_{n}>0$ for large $n$, we have

$$
\begin{aligned}
& 1+u_{n}=1+t_{n}^{-1}\left(\sqrt{\theta_{1}} \phi_{1}+u_{n}^{\top}\right)>0, \\
& 1+v_{n}=1+t_{n}^{-1}\left(\sqrt{\theta_{2}} \phi_{1}+v_{n}^{\top}\right)>0 .
\end{aligned}
$$

Then since $t_{n} \rightarrow 0$ as $n \rightarrow \infty$ and $t_{n}>0$, the following pointwise estimates hold in $\Omega$ as $n \rightarrow \infty$ :

$$
\begin{aligned}
& \left(t_{n}+\sqrt{\theta_{1}} \phi_{1}+u_{n}^{\top}\right)^{-\alpha_{1}} \phi_{1} \rightarrow\left(\theta_{1}\right)^{-\alpha_{1} / 2} \phi_{1}^{1-\alpha_{1}}<2\left(\theta_{1}\right)^{-\alpha_{1} / 2} \phi_{1}^{1-\alpha_{1}}, \\
& \left(t_{n}+\sqrt{\theta_{2}} \phi_{1}+v_{n}^{\top}\right)^{-\alpha_{1}} \phi_{1} \rightarrow\left(\theta_{2}\right)^{-\alpha_{1} / 2} \phi_{1}^{1-\alpha_{1}}<2\left(\theta_{2}\right)^{-\alpha_{1} / 2} \phi_{1}^{1-\alpha_{1}} .
\end{aligned}
$$

Consequently, using (H4+), we get the following pointwise estimate a.e. in $\Omega$ for sufficiently large $n$ :

$$
\begin{aligned}
& g\left(\lambda_{n}, x, u_{n}\right)\left(1+t_{n}^{-1}\left(\sqrt{\theta_{1}} \phi_{1}+u_{n}^{\top}\right)\right)^{\alpha_{1}}\left(t_{n}+\sqrt{\theta_{1}} \phi_{1}+u_{n}^{\top}\right)^{-\alpha_{1}} \phi_{1} \\
& \quad=g\left(\lambda_{n}, x, u_{n}\right)\left(1+u_{n}\right)^{\alpha_{1}}\left(t_{n}+\sqrt{\theta_{1}} \phi_{1}+u_{n}^{\top}\right)^{-\alpha_{1}} \phi_{1} \\
& \quad \geq-2\left(\theta_{1}\right)^{-\alpha_{1} / 2} B_{1} \phi_{1}^{1-\alpha_{1}} .
\end{aligned}
$$

Similarly, for sufficiently large $n$

$$
\begin{aligned}
& f\left(\lambda_{n}, x, v_{n}\right)\left(1+t_{n}^{-1}\left(\sqrt{\theta_{2}} \phi_{1}+v_{n}^{\top}\right)\right)^{\alpha_{1}}\left(t_{n}+\sqrt{\theta_{2}} \phi_{1}+v_{n}^{\top}\right)^{-\alpha_{1}} \phi_{1} \\
& \quad \geq-2\left(\theta_{2}\right)^{-\alpha_{1} / 2} B_{1} \phi_{1}^{1-\alpha_{1}} .
\end{aligned}
$$

Observe that there exist $c_{1}, c_{2}>0$, and $\delta>0$, such that

$$
c_{1} \operatorname{dist}(x, \partial \Omega) \leq \phi_{1}(x) \leq c_{2} \operatorname{dist}(x, \partial \Omega) ; \quad x \in \Omega \quad \text { and } \quad \operatorname{dist}(x, \partial \Omega)<\delta .
$$

Therefore, since $B_{1} \in L^{r}(\Omega)$ and $0 \leq \alpha_{1}<2-\frac{1}{r}$, the Hölder inequality asserts $B_{1} \phi_{1}^{1-\alpha_{1}} \in$ $L^{1}(\Omega)$ (see [1] for a detailed discussion). The estimates (4.10) and (4.11) allow for the use 
of Fatou's lemma below yielding

$$
\begin{aligned}
& \liminf _{n \rightarrow+\infty} \int_{\Omega}\left(\sqrt{\theta_{2}} f\left(\lambda_{n}, x, v_{n}\right)+\sqrt{\theta_{1}} g\left(\lambda_{n}, x, u_{n}\right)\right) \phi_{1} \mathrm{~d} x \\
&=\liminf _{n \rightarrow+\infty} \int_{\Omega}\left[\sqrt{\theta_{2}} f\left(\lambda_{n}, x, v_{n}\right)\left(1+t_{n}^{-1}\left(\sqrt{\theta_{2}} \phi_{1}+v_{n}^{\top}\right)\right)^{\alpha_{1}}\left(1+t_{n}^{-1}\left(\sqrt{\theta_{2}} \phi_{1}+v_{n}^{\top}\right)\right)^{-\alpha_{1}} \phi_{1}\right. \\
&\left.\quad+\sqrt{\theta_{1}} g\left(\lambda_{n}, x, u_{n}\right)\left(1+t_{n}^{-1}\left(\sqrt{\theta_{1}} \phi_{1}+u_{n}^{\top}\right)\right)^{\alpha_{1}}\left(1+t_{n}^{-1}\left(\sqrt{\theta_{1}} \phi_{1}+u_{n}^{\top}\right)\right)^{-\alpha_{1}} \phi_{1}\right] \mathrm{d} x \\
&=\liminf _{n \rightarrow+\infty} \int_{\Omega} t_{n}^{\alpha_{1}}\left[\sqrt{\theta_{2}} f\left(\lambda_{n}, x, v_{n}\right)\left(1+t_{n}^{-1}\left(\sqrt{\theta_{2}} \phi_{1}+v_{n}^{\top}\right)\right)^{\alpha_{1}}\left(t_{n}+\sqrt{\theta_{2}} \phi_{1}+v_{n}^{\top}\right)^{-\alpha_{1}} \phi_{1}\right. \\
&\left.\quad+\sqrt{\theta_{1}} g\left(\lambda_{n}, x, u_{n}\right)\left(1+t_{n}^{-1}\left(\sqrt{\theta_{1}} \phi_{1}+u_{n}^{\top}\right)\right)^{\alpha_{1}}\left(t_{n}+\sqrt{\theta_{1}} \phi_{1}+u_{n}^{\top}\right)^{-\alpha_{1}} \phi_{1}\right] \mathrm{d} x \\
& \stackrel{\text { (i) } x}{\geq} \int_{\Omega} \liminf _{n \rightarrow+\infty} t_{n}^{\alpha_{1}}\left[\sqrt{\theta_{2}} f\left(\lambda_{n}, x, v_{n}\right)\left(1+t_{n}^{-1}\left(\sqrt{\theta_{2}} \phi_{1}+v_{n}^{\top}\right)\right)^{\alpha_{1}}\right. \\
&\left.\quad+\sqrt{\theta_{1}} g\left(\lambda_{n}, x, u_{n}\right)\left(1+t_{n}^{-1}\left(\sqrt{\theta_{1}} \phi_{1}+u_{n}^{\top}\right)\right)^{\alpha_{1}}\right] \phi_{1}^{1-\alpha_{1}} \mathrm{~d} x \\
& \geq \int_{\Omega} \liminf _{(\lambda, s) \rightarrow\left(v_{1},+\infty\right)}\left[\sqrt{\theta_{2}} f(\lambda, x, s)(1+s)^{\alpha_{1}}+\sqrt{\theta_{1}} g(\lambda, x, s)(1+s)^{\alpha_{1}}\right] \phi_{1}^{1-\alpha_{1}} \mathrm{~d} x \\
& \stackrel{(\mathrm{ii})}{=} \int_{\Omega} \liminf _{(\lambda, s) \rightarrow\left(v_{1},+\infty\right)}\left[\sqrt{\theta_{2}} f(\lambda, x, s) s^{\alpha_{1}}+\sqrt{\theta_{1}} g(\lambda, x, s) s^{\alpha_{1}}\right] \phi_{1}^{1-\alpha_{1}} \mathrm{~d} x \\
&= \int_{\Omega} \underline{A}_{\alpha_{1}}^{+} \phi_{1}^{1-\alpha_{1}} \mathrm{~d} x \stackrel{\text { (iii) }}{>} 0 .
\end{aligned}
$$

The inequality (i) follows from Fatou's lemma, (ii) follows since

$$
\begin{aligned}
& \liminf _{(\lambda, s) \rightarrow\left(\nu_{1},+\infty\right)} f(\lambda, x, s)(1+s)^{\alpha_{1}} \\
& =\liminf _{(\lambda, s) \rightarrow\left(\nu_{1},+\infty\right)} f(\lambda, x, s) s^{\alpha_{1}} \lim _{s \rightarrow+\infty} \frac{(1+s)^{\alpha_{1}}}{s^{\alpha_{1}}} \\
& =\liminf _{(\lambda, s) \rightarrow\left(\nu_{1},+\infty\right)} f(\lambda, x, s) s^{\alpha_{1}},
\end{aligned}
$$

and the last inequality follows from assumption (1.5). The inequality (iii) is a contradiction to (4.9). Therefore, for $t_{n}>0, v_{1}-\varepsilon \leq \lambda_{n}<v_{1}$ for large $n$ by (4.8). This means that there is no positive solution for $v_{1}<\lambda_{n} \leq v_{1}+\varepsilon$ for large $n$ and thus part (I) assertion of Theorem 1.2 holds for positive solutions.

Proof of part (II) for positive solutions: Now it follows from Theorem 1.1 and part (I) above that the continuum $\mathscr{D}_{v_{1}}^{+} \subset \mathscr{T}$, from Theorem 1.1, bifurcates from infinity at $v_{1}$ to the left. This concludes the proof of Theorem 1.2 for positive solutions.

Proof of Theorem 1.2 for negative solutions: The proof for negative solutions can be carried out in a similar fashion by using $t_{n}<0$, defining

$$
\begin{aligned}
& -1+u_{n}=-1+t_{n}^{-1}\left(\sqrt{\theta_{1}} \phi_{1}+u_{n}^{\top}\right)<0, \\
& -1+v_{n}=-1+t_{n}^{-1}\left(\sqrt{\theta_{2}} \phi_{1}+v_{n}^{\top}\right)<0,
\end{aligned}
$$

and using $-|s|^{\alpha_{2}}$ instead of $s^{\alpha_{1}}$ in applying Fatou's lemma using (H4-) and reversing the inequalities appropriately.

Proof of Theorem 1.3 The proof of Theorem 1.3 is similar with obvious changes. 
Remark 4.1 In [1], for the scalar case, the integral is analyzed for definite sign using $\|u\|_{W^{2, r}(\Omega)}$ as a parameter. For the case of systems, we use $t^{-1}$ instead of the norm of $\|(u, v)\|_{E}$, which allows us to analyze crucial integrals using only the parameter $t^{-1}$.

\section{Proofs of Theorems 1.4-1.5 and Corollaries 1.6-1.7}

Proof of Theorem 1.4 By Theorem 1.2, it follows that for $\lambda \in\left[v_{1}-\varepsilon, v_{1}\right)$ there exist a positive solution on the continua $\mathscr{D}_{\nu_{1}}^{+}$and a negative solution on the continua $\mathscr{D}_{v_{1}}^{-}$. This proves part (a).

For part (b), first we establish the result for the non-resonant case. For this, define $T_{\lambda}$ : $L^{r}(\Omega) \times L^{r}(\Omega) \rightarrow E$ to be the solution operator of

$$
\left.\begin{array}{ll}
-\Delta u-\lambda \theta_{1} a(x) v=\psi_{1}(x) & \text { in } \Omega, \\
-\Delta v-\lambda \theta_{2} a(x) u=\psi_{2}(x) & \text { in } \Omega, \\
u=0=v \quad \text { on } \partial \Omega . &
\end{array}\right\}
$$

For $\lambda \neq v_{k}$, by the Fredholm alternative, the above system has a unique strong solution for any $\left(\psi_{1}, \psi_{2}\right) \in L^{r}(\Omega) \times L^{r}(\Omega)$. Therefore $\operatorname{Dom}\left(T_{\lambda}\right)=L^{r}(\Omega) \times L^{r}(\Omega)$ and $T_{\lambda}$ is continuous. Then it follows from the standard compactness argument that $T_{\lambda}$ is compact. Next, define $N_{\lambda}: E \rightarrow L^{r}(\Omega) \times L^{r}(\Omega)$ by $N_{\lambda}(u, v)=(f(\lambda, x, v), g(\lambda, x, u))$. Then the operator equation corresponding to (1.1) is

$$
(u, v)=T_{\lambda} N_{\lambda}(u, v)
$$

Now we show that for each fixed $\lambda \neq v_{k}$, for any $|k| \in \mathbb{N}$, there exists a constant $c(\lambda)>0$ such that $\|(u, v)\|_{E} \leq c(\lambda)$. Indeed, suppose to the contrary that $\left\|\left(u_{n}, v_{n}\right)\right\|_{E} \rightarrow \infty$ as $n \rightarrow \infty$. Since $f$ and $g$ satisfy (H3), it follows from (C.1) that

$$
\frac{\left\|N_{\lambda}\left(u_{n}, v_{n}\right)\right\|_{L^{r}(\Omega) \times L^{r}(\Omega)}}{\left\|\left(u_{n}, v_{n}\right)\right\|_{E}} \rightarrow 0 \quad \text { as } n \rightarrow \infty .
$$

Dividing (5.1) by $\left\|\left(u_{n}, v_{n}\right)\right\|_{E}$ and taking the $E$ norm yields

$$
1 \leq \frac{\left\|T_{\lambda}\right\|\left\|N_{\lambda}\left(u_{n}, v_{n}\right)\right\|_{E}}{\left\|\left(u_{n}, v_{n}\right)\right\|_{E}} \rightarrow 0 \quad \text { as } n \rightarrow \infty,
$$

which is absurd. Then by the Schauder fixed point theorem, (5.1) has a solution for each $\lambda \neq v_{k}$ for any $|k| \in \mathbb{N}$ and hence for (1.1). In particular, (1.1) has at least one solution for all $\lambda \in\left(v_{1}, v_{1}+\varepsilon\right]$.

To complete the proof of part (b), it remains to show that (1.1) has a solution for $\lambda=v_{1}$. Theorem 1.1 implies that any solution $(\lambda,(u, v))$ of $(1.1)$ with $\lambda \in\left[v_{1}-\varepsilon, v_{1}+\varepsilon\right]$ and $\|(u, v)\|_{E}>K$ are either $u>0, v>0$ or $u<0, v<0$ in $\Omega$. But part (I) of Theorem 1.2 implies that there are no positive or negative solutions $(\lambda,(u, v)) \in \mathscr{T}$ with $\lambda \in\left[v_{1}, v_{1}+\varepsilon\right]$ and $\|(u, v)\|_{E}>K$. Therefore all solutions $(\lambda,(u, v))$ with $\lambda \in\left[v_{1}, v_{1}+\varepsilon\right]$ must satisfy the uniform bound $\|(u, v)\|_{E} \leq K$.

Now let $\left(\lambda_{n},\left(u_{n}, v_{n}\right)\right)$ be a sequence of solutions of (1.1) with $\lambda_{n} \in\left(v_{1}, v_{1}+\varepsilon\right]$ such that $\lambda_{n} \searrow v_{1}$. Then for each $n \in \mathbb{N},\left(u_{n}, v_{n}\right)$ satisfy

$$
\left.\begin{array}{ll}
u_{n}=\left(-\Delta^{-1}\right)\left(\lambda_{n} \theta_{1} a(x) v_{n}+f\left(\lambda_{n}, x, v_{n}\right)\right) & \text { in } \Omega, \\
v_{n}=\left(-\Delta^{-1}\right)\left(\lambda_{n} \theta_{2} a(x) u_{n}+g\left(\lambda_{n}, x, u_{n}\right)\right) & \text { in } \Omega, \\
u_{n}=0=v_{n} \quad \text { on } \partial \Omega . &
\end{array}\right\}
$$


Since $\left\|\left(u_{n}, v_{n}\right)\right\|_{E} \leq K$, independent of $n$, there exists a subsequence, denoted again by $\left(u_{n}, v_{n}\right)$, that converges to some $(u, v)$ in $C^{1, \eta}(\bar{\Omega}) \times C^{1, \eta}(\bar{\Omega})$ for some $\eta \in(0,1)$. Then $f\left(\lambda_{n}, \cdot, v_{n}\right) \rightarrow f(\lambda, \cdot, v)$ and $g\left(\lambda_{n}, \cdot, u_{n}\right) \rightarrow g(\lambda, \cdot, u)$ in $L^{r}(\Omega)(c f$. [15, Theorem 2.3]). Thus the right-hand sides of the above system converges in $L^{r}(\Omega)$. By the same argument as in the proof of Proposition 3.1, $\left(u_{n}, v_{n}\right) \rightarrow(u, v)$ in $E$ and the limit $(u, v)$ satisfies

$$
\begin{aligned}
& u=\left(-\Delta^{-1}\right)\left(v_{1} \theta_{1} a(x) v+f(\lambda, x, v)\right) \quad \text { in } \Omega, \\
& v=\left(-\Delta^{-1}\right)\left(v_{1} \theta_{2} a(x) u+g(\lambda, x, u)\right) \quad \text { in } \Omega \text {, } \\
& u=0=v \quad \text { on } \partial \Omega \text {, }
\end{aligned}
$$

that is, it satisfies (1.1) for $\lambda=v_{1}$. This establishes the existence of a solution in the resonant case. Thus the proof of Theorem 1.4 is complete.

The proof of Theorem 1.5 follows similarly with appropriate changes.

Proof of Corollary 1.6 We will use Theorem 1.4 for $\alpha_{1}=0=\alpha_{2}$ with $f(\lambda, x, v)=\tilde{f}(v)+h_{1}(x)$ and $g(\lambda, x, u)=\tilde{g}(u)+h_{2}(x)$. Then it is easy to see that hypothesis (1.7) is satisfied by (1.10) with

$$
\begin{aligned}
& \underline{A}_{0}^{+}=\sqrt{\theta_{2}}\left(\tilde{f}_{+\infty}+h_{1}(x)\right)+\sqrt{\theta_{1}}\left(\tilde{g}_{+\infty}+h_{2}(x)\right) \text { and } \\
& \bar{A}_{0}^{-}=\sqrt{\theta_{2}}\left(\tilde{f}_{-\infty}+h_{1}(x)\right)+\sqrt{\theta_{1}}\left(\tilde{g}_{-\infty}+h_{2}(x)\right) .
\end{aligned}
$$

Therefore, by Theorem 1.4, the system (1.9) has a solution.

The proof of Corollary 1.7 follows similarly with appropriate changes.

\section{Examples}

In this section we provide several examples of nonlinear perturbations $f$ and $g$ satisfying the hypotheses of our theorems. All examples below satisfy the hypotheses of Theorem 1.1.

Example 6.1 (Unbounded perturbation: semipositone case) Let $0<\beta_{1} \leq \beta_{2}<1$.

Then $f(\lambda, s)=\lambda\left(|s|^{\beta_{1}}-1\right)$ and $g(\lambda, s)=\lambda\left(|s|^{\beta_{2}}-1\right)$ satisfy

$$
\liminf _{(\lambda, s) \rightarrow\left(\mu_{1},+\infty\right)} \lambda\left(\sqrt{\theta_{2}}|s|^{\beta_{1}}+\sqrt{\theta_{1}}|s|^{\beta_{2}}-\sqrt{\theta_{2}}-\sqrt{\theta_{1}}\right)=+\infty>0 .
$$

Thus the hypotheses of Theorem 1.2 are satisfied for $\alpha=0$.

Example 6.2 (Bounded perturbation: semipositone case) Let $0<\beta_{1} \leq \beta_{2}$. Then $f(\lambda, s)=-\lambda\left[1 /\left(1+|s|^{\beta_{1}}\right)+2\right]$ and $g(\lambda, s)=-\lambda\left[1 /\left(1+|s|^{\beta_{2}}\right)+2\right]$ satisfy

$$
\limsup _{(\lambda, s) \rightarrow\left(\mu_{1},+\infty\right)}-\lambda\left(\frac{\sqrt{\theta_{2}}}{1+|s|^{\beta_{1}}}+\frac{\sqrt{\theta_{1}}}{1+|s|^{\beta_{2}}}+2\left(\sqrt{\theta_{1}}+\sqrt{\theta_{2}}\right)\right)=-2\left(\sqrt{\theta_{1}}+\sqrt{\theta_{2}}\right) \mu_{1}<0 .
$$

Thus the hypotheses of Theorem 1.3 are satisfied for $\alpha=0$.

Example 6.3 (Vanishing perturbation) Let $N=2,0<\varepsilon_{1} \leq \varepsilon_{2}<3 / 2$. 
Then $f(s)=1 /\left(1+|s|^{3 / 2-\varepsilon_{1}}\right)$ and $g(s)=1 /\left(1+|s|^{3 / 2-\varepsilon_{2}}\right)$ satisfy

$$
\liminf _{(\lambda, s) \rightarrow\left(\mu_{1},+\infty\right)}\left[\frac{\sqrt{\theta_{2}}}{1+s^{3 / 2-\varepsilon_{1}}}+\frac{\sqrt{\theta_{1}}}{1+s^{3 / 2-\varepsilon_{2}}}\right](1+s)^{3 / 2-\varepsilon_{1}} \geq \sqrt{\theta_{2}}>0 .
$$

Thus the hypotheses of Theorem 1.2 are satisfied.

Example 6.4 (Oscillating perturbation) Let $0<\beta<1$ and $0<\gamma$.

Then $f(\lambda, s)=(2+\sin (s))|\lambda s|^{\beta}$ and $g(\lambda, s)=\cos \left(\lambda s^{2}\right) /\left(1+|s|^{\gamma}\right)$ satisfy

$$
\liminf _{(\lambda, s) \rightarrow\left(\mu_{1},+\infty\right)}\left[\sqrt{\theta_{2}}(2+\sin (s))|\lambda s|^{\beta}+\frac{\sqrt{\theta_{1}} \cos \left(\lambda s^{2}\right)}{1+s^{\gamma}}\right]=+\infty>0
$$

Thus the hypotheses of Theorem 1.2 are satisfied for $\alpha=0$.

Example 6.5 (Landesman-Lazer type perturbation) Let $a(x) \equiv 1, \tilde{f}(s)=\arctan (s)$, and $\tilde{g}(s)=-\arctan (s)$. Then

(i) if $\theta_{1}<\theta_{2}$ then (1.9) has a solution provided $h_{1}, h_{2} \in L^{r}(\Omega)$ satisfies (1.10), and

(ii) if $\theta_{1}>\theta_{2}$ then (1.9) has a solution provided $h_{1}, h_{2} \in L^{r}(\Omega)$ satisfies (1.11).

\section{Appendix 1: Abstract bifurcation}

We shall use results from the abstract bifurcation theory to prove our existence results. In particular, the theory of a bifurcation from infinity was developed mainly by Rabinowitz [17]. It is well known that the result on a bifurcation from infinity [17] is developed from the theory of a bifurcation from zero [18]. However, it was pointed out by Dancer [19] that the proofs of Theorem 1.27 and Theorem 1.40 in [18] contain gaps. Moreover, as pointed out in [20], the results from [18] for a bifurcation from zero are also wrong in their statement. Thus the statement and the proof of the result on a bifurcation from infinity [17] should be revisited in the spirit of discussion in [19-21]. We were not able to find these corrected statements and proofs for a bifurcation from infinity anywhere in the literature. Thus we restate and prove a corrected version of [17, Theorem 1.8] for the sake of the readers.

In their abstract setting, the bifurcations are studied in the product space $\mathbb{R} \times \mathscr{B}$ where $\mathscr{B}$ is a real Banach space with norm $\|\cdot\|_{\mathscr{B}}$. For $(\lambda, z) \in \mathbb{R} \times \mathscr{B}$, we consider the norm $\|(\lambda, z)\|_{\mathbb{R} \times \mathscr{B}}:=|\lambda|+\|z\|_{\mathscr{B}}$. In what follows, by connected components of a topological space we mean the maximal connected subsets (ordered by inclusion) of the given space. We use the abstract bifurcation theorems concerning the bifurcation of continua of solutions from infinity for the operator equation

$$
z=\lambda L z+H(\lambda, z)
$$

Here $\lambda \in \mathbb{R}$ is the bifurcation parameter and $L: \mathscr{B} \rightarrow \mathscr{B}$ is a linear and compact operator, $H: \mathbb{R} \times \mathscr{B} \rightarrow \mathscr{B}$ is a continuous and compact operator which satisfies sublinearity condition at infinity, that is,

$$
\lim _{\|z\|_{\mathscr{B}} \rightarrow+\infty} \frac{\|H(\lambda, z)\|_{\mathscr{B}}}{\|z\|_{\mathscr{B}}}=0
$$

uniformly on compact $\lambda$-intervals. 
It was shown by Krasnosel'skii [16, p.194] that a necessary condition for $(\mu,+\infty)$ to be a bifurcation point from infinity is that $\mu$ is a characteristic eigenvalue of $L$.

$$
\begin{aligned}
& \text { Define }{ }_{0} H: \mathbb{R} \times \mathscr{B} \rightarrow \mathscr{B} \\
& { }_{0} H(\lambda, w):= \begin{cases}\|w\|_{\mathscr{B}}^{2} H\left(\lambda, w /\|w\|_{\mathscr{B}}^{2}\right) ; & w \neq 0, \\
0 ; & w=0 .\end{cases}
\end{aligned}
$$

Assume in addition that

- ${ }_{0} H: \mathbb{R} \times \mathscr{B} \rightarrow \mathscr{B}$ is compact,

as continuity follows from (A.2). Let $\mu \in \mathbb{R}$ be a characteristic value of $L$ of odd multiplicity. It was proved in [17, Theorem 1.6] that the set

$$
\mathscr{T}=\{(\lambda, z) \in \mathbb{R} \times \mathscr{B}: \text { Eq. (A.1) holds }\}
$$

possesses an unbounded component (continuum) $\mathscr{D} \subset \mathscr{T}$ which meets $(\mu, \infty)$ and satisfies the dichotomy of $[17$, Theorem 1.6]. In other words, $\mathscr{D}$ meets $\{(\lambda, 0): \lambda \in \mathbb{R}\}$, or meets $(\hat{\mu},+\infty)$ where $\hat{\mu}_{\infty} \neq \mu_{\infty}$ is another characteristic value of odd multiplicity, or $\mathscr{D}$ is unbounded in the $\lambda$-direction.

This abstract result for a bifurcation from infinity can easily be strengthened by using $[19$, Theorem 2], which applies to a bifurcation from zero from a characteristic value $\mu \in \mathbb{R}$ of multiplicity 1 . For $\varepsilon>0$, define

$$
\mathcal{O}_{\varepsilon}(\mu):=\left\{(\lambda, z) \in \mathbb{R} \times \mathscr{B}:\|(\lambda, z)\|_{\mathbb{R} \times \mathscr{B}}=|\lambda-\mu|+1 /\|z\|_{\mathscr{B}}<\varepsilon\right\} .
$$

We now state the correct result as intended in [17, Theorem 1.8] and provide the proof below.

Proposition A.1 Let $L, H$, and ${ }_{0} H$ be as above. Let $\mu$ be a characteristic value of $L$ of multiplicity 1 , and let $\vartheta \in \mathscr{B}$ satisfying $\vartheta \neq 0$ and $\mu L \vartheta=\vartheta$ be fixed. Then there exist $K>0$ and two continua $\mathscr{D}^{v}, v= \pm$, of solutions to (A.1) such that for all $0<\varepsilon \leq K: \mathscr{D}^{v} \cap \mathcal{O}_{\varepsilon} \neq \emptyset$. Moreover, $(\lambda, u) \in \mathscr{D}^{v} \cap \mathcal{O}_{K}$ implies $(\lambda, u)=(\lambda, \tau \vartheta+\omega)$ with $\tau \in \mathbb{R}: v \tau>0, \omega \in \mathscr{B}$, and $|\lambda-\mu| \rightarrow 0$ and $\|\omega\|_{\mathscr{B}} /|\tau| \rightarrow 0$ as $|\tau| \rightarrow \infty$.

Proof Indeed, bifurcations from infinity can be studied via bifurcations from zero using the Kelvin transform $z \mapsto z /\|z\|_{\mathscr{B}}^{2}$ for $\|z\|_{\mathscr{B}} \neq 0$, which turns solutions large in the norm to solutions small in the norm and vice versa. For $z \neq 0$ we set $w:=z /\|z\|_{\mathscr{B}}^{2}$. Note that $z=w /\|w\|_{\mathscr{B}}^{2}$ for $w \neq 0$, reciprocally, and $\|z\|_{\mathscr{B}}=1 /\|w\|_{\mathscr{B}}$ for $w \neq 0$. Now dividing (A.1) by $\|z\|_{\mathscr{B}}^{2} \neq 0$ we obtain $w=\lambda L w+\|w\|^{2} H\left(\lambda, w /\|w\|_{\mathscr{B}}^{2}\right)$. Since ${ }_{0} H$ is continuous and compact, the equation

$$
w=\lambda L w+{ }_{0} H(\lambda, w)
$$

satisfies the assumptions of [19, Theorem 2], which we describe briefly below for clarity and completeness of the proof. For this, we borrow the notation from [19]. Let $\mu \in \mathbb{R}$ be a characteristic value of $L$ of multiplicity $1, \vartheta \in \mathscr{B} \backslash\{0\}$, and $l \in \mathscr{B}^{*} \backslash\{0\}$ satisfy $\vartheta=\mu L \vartheta$ 
and $l=\mu L^{*} l$ (where $L^{*}$ is adjoint to $L$ ) and $l(\vartheta)=1$. For $0<y<1$, define

$$
\begin{aligned}
& K_{y}:=\left\{(\lambda, z) \in \mathbb{R} \times \mathscr{B}:|l(z)|>y\|z\|_{\mathscr{B}}\right\} \\
& K_{y}^{ \pm}:=\left\{(\lambda, z) \in \mathbb{R} \times \mathscr{B}: \pm l(z)>y\|z\|_{\mathscr{B}}\right\} .
\end{aligned}
$$

In particular, $K_{y}^{v}, v=+,-$, are convex cones, $K_{y}^{+}=-K_{y}^{+}$, and $K_{y}^{v}=K_{y} \backslash K_{y}^{-v}$, where $-v$ stands for the sign opposite to $v$. Let $\mathscr{S}$ denote the closure of all nontrivial solutions of (A.3) in $\mathbb{R} \times \mathscr{B}$, that is,

$$
\mathscr{S}:=\overline{\{(\lambda, w) \in \mathbb{R} \times \mathscr{B}:(\lambda, w) \text { satisfies }(\mathrm{A} .3) \text { and } w \neq 0\}}
$$

and $\mathscr{S}_{\mu}$ denote the component of $\mathscr{S}$ such that $(0, \mu) \in \mathscr{S}_{\mu}$.

For any $0<y<1$, by [18, Lemma 1.24], there exists $S>0$ such that

$$
(\mathscr{S} \backslash\{(\mu, 0)\}) \cap \overline{\mathcal{E}}_{S}(\mu) \subset K_{y} .
$$

Then for $0<\varepsilon \leq S$ define

$$
\mathcal{E}_{\varepsilon}(\mu):=\left\{(\lambda, z) \in \mathbb{R} \times \mathscr{B}:\|(\lambda, z)\|_{\mathbb{R} \times \mathscr{B}}=|\lambda-\mu|+\|z\|_{\mathscr{B}}<\varepsilon\right\}
$$

and for $v= \pm$ define $\mathcal{D}_{\mu, \varepsilon}^{v}$ to be the component of $\{(\mu, 0)\} \cup\left(\mathscr{S} \cap \overline{\mathcal{E}}_{\varepsilon}(\mu) \cap K_{\mu}^{v}\right)$ containing $(\mu, 0)$. Let $\mathscr{C}_{\mu, \varepsilon}^{\nu}$ be the component of $\overline{\mathscr{S}_{\mu} \backslash \mathcal{D}_{\mu, \varepsilon}^{-v}}$ containing $(\mu, 0)$ (here again $-v=\mp$ for $v= \pm$ ). Finally, we define $\mathscr{C}_{\mu}^{\nu}$ to be the closure of $\bigcup_{0 \leq \varepsilon<S} \mathscr{C}_{\mu, \varepsilon}^{\nu}$ in $\mathbb{R} \times \mathscr{B}$. Then by [19, Theorem 2] the following dichotomy holds:

(i) either $\mathscr{C}_{\mu}^{+}$and $\mathscr{C}_{\mu}^{-}$are both unbounded,

(ii) or $\mathscr{C}_{\mu}^{+} \cap \mathscr{C}_{\mu}^{-} \neq\{(\mu, 0)\}$.

Now fix $0<y<1$. Then for all $0<\varepsilon \leq S$, one has

$$
(\mathscr{S} \backslash\{(\mu, 0)\}) \cap \overline{\mathcal{E}}_{\varepsilon}(\mu) \subset K_{y},
$$

by (A.4). By the respective definitions of $\mathscr{C}_{\mu}^{\nu}$ where $v= \pm$, we see that for all $0<\varepsilon \leq S$,

$$
\left(\mathscr{C}_{\mu}^{\nu} \backslash\{(\mu, 0)\}\right) \cap \overline{\mathcal{E}}_{\varepsilon}(\mu) \subset K_{y}^{v}
$$

Now let us assume that $\mathscr{C}_{\mu}^{+}$and $\mathscr{C}_{\mu}^{-}$are both unbounded. Then they must leave the bounded sets $\overline{\mathcal{E}}_{S}(\mu) \cap K_{y}^{v}$ through $\partial \overline{\mathcal{E}}_{S}(\mu) \cap K_{y}^{v}$, and thus the intersections $\mathscr{C}_{\mu}^{v} \cap \partial \overline{\mathcal{E}}_{S}(\mu) \cap K_{y}^{v}$ where $v= \pm$ are nonempty. If at least one of $\mathscr{C}_{\mu}^{+}$or $\mathscr{C}_{\mu}^{-}$is bounded, then, by the second part of the dichotomy, we have $\mathscr{C}_{\mu}^{+} \cap \mathscr{C}_{\mu}^{-} \neq\{(\mu, 0)\}$. This entails that both continua $\mathscr{C}_{\mu}^{v}$ must leave $\overline{\mathcal{E}}_{S}(\mu) \cap K_{y}^{v}$ through $\partial \overline{\mathcal{E}}_{S}(\mu) \cap K_{y}^{v}$. This shows that in both cases of the dichotomy $\mathscr{C}_{\mu}^{v} \cap \partial \overline{\mathcal{E}}_{S}(\mu) \cap K_{y}^{v} \neq \emptyset$. It is worth noting that each component of the set $\mathscr{C}_{\mu}^{v} \cap \overline{\mathcal{E}}_{S}(\mu) \cap K_{y}^{v}$ has a nonempty intersection with the set $\partial \overline{\mathcal{E}}_{S}(\mu) \cap K_{y}^{v}$ but there may exist components that do not have $(\mu, 0)$ in their closure. For each $v= \pm$, there exists a component of $\mathscr{C}_{\mu}^{v} \cap \overline{\mathcal{E}}_{S}(\mu) \cap K_{y}^{v} \backslash\{(\mu, 0)\}$, denoted by $\mathscr{K}_{\mu}^{\nu}$, such that $\{(\mu, 0)\} \in \overline{\mathscr{K}_{\mu}^{v}}$ and $\mathscr{K}_{\mu}^{v} \cap \partial \overline{\mathcal{E}}_{S}(\mu) \cap K_{y}^{v} \neq \emptyset$. Indeed, it suffices to show the existence of such a component satisfying $\{(\mu, 0)\} \in \overline{\mathscr{K}_{\mu}^{\nu}}$. We prove by contradiction. Suppose there exists $r^{*} \in(0, S)$ such that all components of $\mathscr{C}_{\mu}^{v} \cap \overline{\mathcal{E}}_{S}(\mu) \cap K_{y}^{v} \backslash\{(\mu, 0)\}$ are disjoint from $(\mu, 0)$ by the ball $\mathcal{E}_{r^{*}}$, that 
is, $\mathscr{C}_{\mu}^{v} \cap \overline{\mathcal{E}}_{S}(\mu) \cap K_{y}^{v} \backslash\{(\mu, 0)\} \cap \mathcal{E}_{r^{*}}=\emptyset$. But this is not possible since $(\mu, 0) \in \mathscr{C}_{\mu}^{v} \cap \mathcal{E}_{S}$ and $\mathscr{C}_{\mu}^{v}$ is a closed connected set.

Now let $\mathscr{D}_{\mu}^{ \pm}:=\left\{(\lambda, z) \in \mathbb{R} \times \mathscr{B}:\left(\lambda, z /\|z\|_{\mathscr{B}}^{2}\right) \in \mathscr{K}_{\mu}^{ \pm}\right\}$. Taking $K:=S$ we see that $\mathscr{D}^{+} \cap$ $\mathcal{O}_{K}(\mu) \neq \emptyset$ and $\mathscr{D}^{-} \cap \mathcal{O}_{K}(\mu) \neq \emptyset$. Moreover, it follows from [18, Lemma 1.24] that $(\lambda, u) \in$ $\mathscr{D}^{v} \cap \mathcal{O}_{K}$ implies $(\lambda, u)=(\lambda, \tau \vartheta+\omega)$ with $\tau \in \mathbb{R}: \nu \tau>0, \omega \in \mathscr{B}$, and $|\lambda-\mu| \rightarrow 0$ and $\|\omega\|_{\mathscr{B}} /|\tau| \rightarrow 0$ as $|\tau| \rightarrow \infty$. Since $\{(\mu, 0)\} \in \overline{\mathscr{K}_{\mu}^{ \pm}}$, we find that $\mathscr{D}^{ \pm} \cap \mathcal{O}_{\varepsilon}(\mu) \neq \emptyset$ for any $\varepsilon>0$. This completes the proof.

\section{Appendix 2: Eigenvalue problem}

Let $\mu_{j}$, with $j \in \mathbb{N}$ denote the $j$ th eigenvalue of

$$
-\Delta \phi=\lambda a(x) \phi \quad \text { in } \Omega ; \quad \phi=0 \quad \text { on } \partial \Omega .
$$

With these eigenvalues being ordered, $0<\mu_{1}<\mu_{2} \leq \cdots \leq \mu_{j-1} \leq \mu_{j} \leq \cdots$, let $\phi_{j} \in$ $W_{0}^{1,2}(\Omega)$ denote the eigenfunction corresponding to $\mu_{j}$. By the standard regularity argument, $\phi_{j} \in W_{0}^{1,2}(\Omega) \cap W^{2, r}(\Omega)$, for any $r>N$, and it is a strong solution of (1.2) with $\lambda=\mu_{j}$.

Now we describe the eigenvalues and eigenfunctions of the linear system

$$
\left.\begin{array}{l}
-\Delta w_{1}=\lambda \theta_{1} a(x) w_{2} \quad \text { in } \Omega, \\
-\Delta w_{2}=\lambda \theta_{2} a(x) w_{1} \quad \text { in } \Omega, \\
w_{1}=0=w_{2} \quad \text { on } \partial \Omega,
\end{array}\right\}
$$

associated to problem (1.1). Note that the eigenvalues of (B.1) are the characteristic values of the linear operator $L$ that appears in (2.1). We wish to point out that the eigenvalue problems are well studied in the literature for systems, including a more general case than (B.1). We state and prove the property that is necessary for our analysis. Interested readers will find the following references helpful for general linear eigenvalue problems: [22] for a system of $n$ equations and [23, p.106] for a system of two equations; also see the references therein.

Proposition B.1 The eigenvalues of (B.1) form the following set:

$$
\left\{-\frac{\mu_{j}}{\sqrt{\theta_{1} \theta_{2}}}\right\}_{j=1}^{\infty} \cup\left\{\frac{\mu_{j}}{\sqrt{\theta_{1} \theta_{2}}}\right\}_{j=1}^{\infty}
$$

If we order these eigenvalues such that $v_{l}=-\frac{\mu_{-l}}{\sqrt{\theta_{1} \theta_{2}}}$ for $-l \in \mathbb{N}$ and $v_{l}=\frac{\mu_{l}}{\sqrt{\theta_{1} \theta_{2}}}$ for $l \in \mathbb{N}$, then $\left(-\sqrt{\theta_{1}} \phi_{-l}, \sqrt{\theta_{2}} \phi_{-l}\right)$ for $-l \in \mathbb{N}$ and $\left(\sqrt{\theta_{1}} \phi_{l}, \sqrt{\theta_{2}} \phi_{l}\right)$ for $l \in \mathbb{N}$ are the eigenfunctions corresponding to $v_{l}$, respectively. In particular, $v_{1}=\frac{\mu_{1}}{\sqrt{\theta_{1} \theta_{2}}}$ is the only eigenvalue value such that both components of its corresponding eigenfunction are positive in $\Omega$.

Since the operator associated with the linear part of (1.1) is not self-adjoint, we will be dealing with the eigenfunction of (B.1) as well as of the corresponding adjoint equation.

Remark B.2 It is well known that the eigenvalues of the adjoint equation corresponding to (B.1) are same as that of (B.1) but eigenfunctions are given by $\left(-\sqrt{\theta_{2}} \phi_{-l}, \sqrt{\theta_{1}} \phi_{-l}\right)$ for $-l \in \mathbb{N}$ and $\left(\sqrt{\theta_{2}} \phi_{l}, \sqrt{\theta_{1}} \phi_{l}\right)$ for $l \in \mathbb{N}$. 
Proof Using matrix notation for convenience, the eigenvalue problem (B.1) reads as follows:

$$
-\Delta\left[\begin{array}{l}
w_{1} \\
w_{2}
\end{array}\right]=\lambda a(x)\left[\begin{array}{cc}
0 & \theta_{1} \\
\theta_{2} & 0
\end{array}\right]\left[\begin{array}{l}
w_{1} \\
w_{2}
\end{array}\right] \quad \text { in } \Omega .
$$

Note that $-\sqrt{\theta_{2} \theta_{1}}$ and $\sqrt{\theta_{2} \theta_{1}}$,

$$
\left[\begin{array}{c}
-\sqrt{\theta_{1}} \\
\sqrt{\theta_{2}}
\end{array}\right] \text { and }\left[\begin{array}{c}
\sqrt{\theta_{1}} \\
\sqrt{\theta_{2}}
\end{array}\right]
$$

are respective eigenvalues and eigenvectors of the coefficient matrix. Taking into account the linearity of ' $-\Delta$ ', we infer that

$$
\left[\begin{array}{l}
z_{1} \\
z_{2}
\end{array}\right]=\left[\begin{array}{cc}
-\sqrt{\theta_{1}} & \sqrt{\theta_{1}} \\
\sqrt{\theta_{2}} & \sqrt{\theta_{2}}
\end{array}\right]^{-1}\left[\begin{array}{l}
w_{1} \\
w_{2}
\end{array}\right]
$$

satisfies

$$
-\Delta\left[\begin{array}{l}
z_{1} \\
z_{2}
\end{array}\right]=\lambda a(x)\left[\begin{array}{cc}
-\sqrt{\theta_{1} \theta_{2}} & 0 \\
0 & \sqrt{\theta_{1} \theta_{2}}
\end{array}\right]\left[\begin{array}{l}
z_{1} \\
z_{2}
\end{array}\right] \quad \text { in } \Omega .
$$

The equations of this system are not coupled and it is obvious that $z_{1} \not \equiv 0$ if and only if $-\lambda \sqrt{\theta_{1} \theta_{2}}=\mu_{k}$ and $z_{1}=\phi_{k}$ for some $k \in \mathbb{N}$. On the other hand, $z_{2} \not \equiv 0$ if and only if $\lambda \sqrt{\theta_{1} \theta_{2}}=\mu_{l}$ and $z_{2}=\phi_{l}$ for some $l \in \mathbb{N}$. Therefore, $z_{1} \not \equiv 0$ imply $z_{2} \equiv 0$ and $z_{2} \not \equiv 0$ imply $z_{1} \equiv 0$. Hence, the eigenfunctions of (B.2) corresponding to $v_{k}=-\frac{\mu_{k}}{\sqrt{\theta_{1} \theta_{2}}}$ are

$$
\left[\begin{array}{cc}
-\sqrt{\theta_{1}} & \sqrt{\theta_{1}} \\
\sqrt{\theta_{2}} & \sqrt{\theta_{2}}
\end{array}\right]\left[\begin{array}{c}
\phi_{k} \\
0
\end{array}\right]=\left[\begin{array}{c}
-\sqrt{\theta_{1}} \\
\sqrt{\theta_{2}}
\end{array}\right] \phi_{k}
$$

which is $\left(-\sqrt{\theta_{1}} \phi_{k}, \sqrt{\theta_{2}} \phi_{k}\right)$ in the original notation. Analogously, the eigenfunctions of (B.2) corresponding to $v_{k}=\frac{\mu_{k}}{\sqrt{\theta_{1} \theta_{2}}}$ are $\left(\sqrt{\theta_{1}} \phi_{k}, \sqrt{\theta_{2}} \phi_{k}\right)$. Note that $\phi_{1}$ is the only eigenfunction of (1.2) which does not change sign in $\Omega$ and hence can be normalized to be positive on $\Omega$. Thus $\nu_{1}=\frac{\mu_{1}}{\sqrt{\theta_{1} \theta_{2}}}$ is the only eigenvalue of (B.1) such that both components of its eigenfunction $\left(\sqrt{\theta_{1}} \phi_{1}, \sqrt{\theta_{2}} \phi_{1}\right)$ are positive in $\Omega$.

\section{Appendix 3: Proof of (2.2)}

Proof of (2.2) It is enough to show that

$$
\frac{\left\|f\left(\lambda, \cdot, v_{n}\right)\right\|_{L^{r}(\Omega)}}{\left\|\left(u_{n}, v_{n}\right)\right\|_{E}} \rightarrow 0, \quad \frac{\left\|g\left(\lambda, \cdot, u_{n}\right)\right\|_{L^{r}(\Omega)}}{\left\|\left(u_{n}, v_{n}\right)\right\|_{E}} \rightarrow 0
$$

as $n \rightarrow+\infty$ uniformly for $\lambda$ in compact intervals for any sequence $\left(u_{n}, v_{n}\right) \in E$ such that $\left\|\left(u_{n}, v_{n}\right)\right\|_{E} \rightarrow+\infty$. We will show the convergence of the first sequence and the proof of the second is identical. Let $\Lambda \subset \mathbb{R}$ be a compact interval. It follows from (H2) that

$$
\frac{\left|f\left(\lambda, x, v_{n}\right)\right|}{1+\left|v_{n}\right|} \leq \frac{h(x)+b\left|v_{n}\right|}{1+\left|v_{n}\right|} \leq h(x)+\frac{b\left|v_{n}\right|}{1+\left|v_{n}\right|} \leq h(x)+b .
$$


As in [24], for each $n \in \mathbb{N}$, we define sets

$$
\begin{aligned}
& A_{n}:=\left\{x \in \Omega:\left|v_{n}(x)\right| \leq\left\|\left(u_{n}, v_{n}\right)\right\|_{E}^{1 / 2}\right\}, \\
& B_{n}:=\left\{x \in \Omega:\left|v_{n}(x)\right|>\left\|\left(u_{n}, v_{n}\right)\right\|_{E}^{1 / 2}\right\} .
\end{aligned}
$$

Both sets are measurable and $A_{n} \cup B_{n}=\Omega$ for any $n \in \mathbb{N}$. For a.e. $x \in A_{n}$, we have

$$
\frac{\left|f\left(\lambda, x, v_{n}\right)\right|}{\left\|\left(u_{n}, v_{n}\right)\right\|_{E}}=\frac{\left|f\left(x, v_{n}\right)\right|}{1+\left|v_{n}\right|} \cdot \frac{1+\left|v_{n}\right|}{\left\|\left(u_{n}, v_{n}\right)\right\|_{E}}
$$

On the other hand, by the definition of $A_{n}$

$$
\frac{1+\left|v_{n}\right|}{\left\|\left(u_{n}, v_{n}\right)\right\|_{E}} \leq \frac{1+\left\|\left(u_{n}, v_{n}\right)\right\|_{E}^{1 / 2}}{\left\|\left(u_{n}, v_{n}\right)\right\|_{E}}
$$

for a.e. $x \in A_{n}$. Since $\left\|\left(u_{n}, v_{n}\right)\right\|_{E} \rightarrow+\infty$ as $n \rightarrow+\infty$, there exists $n_{0}$ such that $\left\|\left(u_{n}, v_{n}\right)\right\|_{E} \geq$ 1 for all $n>n_{0}$. It follows from (C.6) that

$$
\frac{1+\left|v_{n}\right|}{\left\|\left(u_{n}, v_{n}\right)\right\|_{E}} \leq \frac{2}{\left\|\left(u_{n}, v_{n}\right)\right\|_{E}^{1 / 2}}
$$

for all $n>n_{0}$. Applying (C.2), (C.6), and (C.7) in (C.5) yields

$$
\frac{\left|f\left(\lambda, x, v_{n}\right)\right|}{\left\|\left(u_{n}, v_{n}\right)\right\|_{E}} \leq \frac{2(h(x)+1)}{\left\|\left(u_{n}, v_{n}\right)\right\|_{E}^{1 / 2}}
$$

a.e. in $A_{n}$ and for all $n>n_{0}$. For a.e. $x \in B_{n}$, we have

$$
\left\|\left(u_{n}, v_{n}\right)\right\|_{E}^{1 / 2}<\left|v_{n}\right| \leq\left\|\left(u_{n}, v_{n}\right)\right\|_{\infty} \leq C\left\|\left(u_{n}, v_{n}\right)\right\|_{E},
$$

where $C$ is the constant of the embedding $W^{2, r}(\Omega) \hookrightarrow L^{\infty}(\Omega)$. Then, for $\lambda \in \Lambda$,

$$
\frac{\left|f\left(\lambda, x, v_{n}\right)\right|}{\left\|\left(u_{n}, v_{n}\right)\right\|_{E}} \leq C \frac{\left|f\left(\lambda, x, v_{n}\right)\right|}{\left|v_{n}\right|} \leq C \sup _{|t| \geq\left\|\left(u_{n}, v_{n}\right)\right\|_{E}^{1 / 2}} \frac{|f(\lambda, x,|t|)|}{|t|}
$$

Now let $\chi_{A_{n}}$ and $\chi_{B_{n}}$ denote the characteristic functions of $A_{n}$ and $B_{n}$, respectively. Then, for $\lambda \in \Lambda$,

$$
\begin{aligned}
\frac{\left|f\left(\lambda, x, v_{n}\right)\right|}{\left\|\left(u_{n}, v_{n}\right)\right\|_{E}} & \leq \frac{2(h(x)+1)}{\left\|\left(u_{n}, v_{n}\right)\right\|_{E}^{1 / 2}} \chi_{A_{n}}+C \sup _{|t| \geq\left\|\left(u_{n}, v_{n}\right)\right\|_{E}^{1 / 2}} \frac{|f(\lambda, x,|t|)|}{|t|} \chi_{B_{n}} \\
& \leq \max \left\{\frac{2(h(x)+1)}{\left\|\left(u_{n}, v_{n}\right)\right\|_{E}^{1 / 2}}, C \sup _{|t| \geq\left\|\left(u_{n}, v_{n}\right)\right\|_{E}^{1 / 2}} \frac{|f(\lambda, x,|t|)|}{|t|}\right\} \rightarrow 0
\end{aligned}
$$

for a.e. $x \in \Omega$ since $\sup _{|t| \geq\left\|\left(u_{n}, v_{n}\right)\right\|_{E}^{1 / 2}} \frac{|f(\lambda, x,|t|)|}{|t|} \rightarrow 0$ by (H3). Hence by the Lebesgue dominated convergence theorem ( $\Omega$ is bounded, integrand dominated by 1 ) one can assert that

$$
\lim _{n \rightarrow+\infty} \int_{\Omega}\left|\frac{\left|f\left(\lambda, x, v_{n}\right)\right|}{\left\|\left(u_{n}, v_{n}\right)\right\|_{E}}\right|^{r} \mathrm{~d} x \rightarrow 0
$$


uniformly for $\lambda \in \Lambda$. This establishes (C.1) and hence the proof of the claim is complete.

\section{Competing interests}

The authors declare that they have no competing interests.

\section{Authors' contributions}

Both authors wrote, read, and approved the final manuscript.

\section{Author details}

${ }^{1}$ Department of Mathematics and Statistics, The University of North Carolina at Greensboro, Greensboro, NC 27402, USA

${ }^{2}$ NTIS - New Technologies for the Information Society, European Centre of Excellence, Faculty of Applied Sciences, University of West Bohemia, Univerzitní 8, Plzeň, 30614, Czech Republic. ${ }^{3}$ KMA-FAV, Západočeská Univerzita v Plzni, Univerzitní 22, Plzeň, 30614, Czech Republic.

\section{Acknowledgements}

The second author was supported by the Grant Agency of the Czech Republic Project No. 13-00863S

\section{Received: 20 June 2014 Accepted: 4 November 2014 Published online: 20 November 2014}

\section{References}

1. Arcoya, D, Gámez, JL: Bifurcation theory and related problems: anti-maximum principle and resonance. Commun. Partial Differ. Equ. 26(9-10), 1879-1911 (2001)

2. Ambrosetti, A, Arcoya, D, Buffoni, B: Positive solutions for some semi-positone problems via bifurcation theory. Differ. Integral Equ. 7(3-4), 655-663 (1994)

3. Hai, DD: Singular elliptic systems with asymptotically linear nonlinearities. Differ. Integral Equ. 26(7-8), 837-844 (2013)

4. Hai, DD, Sankar, L, Shivaji, R: Infinite semipositone problems with asymptotically linear growth forcing terms. Differ. Integral Equ. 25(11-12), 1175-1188 (2012)

5. Peng, C: Asymptotically linear elliptic systems with parameters. Glasg. Math. J. 52(2), 383-389 (2010)

6. Peng, C, Yang, J: Positive solutions for asymptotically linear elliptic systems. Glasg. Math. J. 49(2), 377-390 (2007)

7. Mawhin, J, Schmitt, K: Nonlinear eigenvalue problems with the parameter near resonance. Ann. Pol. Math. 51 241-248 (1990)

8. Korman, P: Nonlinear perturbations of linear elliptic systems at resonance. Proc. Am. Math. Soc. 140(7), 2447-2451 (2012)

9. Agudelo, O, Ruf, B, Vélez, C: On a nonlinear elliptic system with symmetric coupling. Nonlinear Anal. 75(11), 4315-4324 (2012)

10. Korman, P: Curves of equiharmonic solutions and solvability of elliptic systems. Differ. Integral Equ. 24(11-12) 1147-1162 (2011)

11. Zuluaga, M: Nonzero solutions of a nonlinear elliptic system at resonance. Nonlinear Anal. 31(3-4), 445-454 (1998)

12. Zuluaga, M: On a nonlinear elliptic system: resonance and bifurcation cases. Comment. Math. Univ. Carol. 40(4), 701-711 (1999)

13. Landesman, EM, Lazer, AC: Nonlinear perturbations of linear elliptic boundary value problems at resonance. J. Math Mech. 19, 609-623 (1969/1970)

14. Gilbarg, D, Trudinger, NS: Elliptic Partial Differential Equations of Second Order. Classics in Mathematics. Springer Berlin (2001). Reprint of the 1998 edition

15. de Figueiredo, DG: Lectures on the Ekeland Variational Principle with Applications and Detours. Tata Institute of Fundamental Research Lectures on Mathematics and Physics, vol. 81. Published for the Tata Institute of Fundamental Research, Bombay. Springer, Berlin (1989)

16. Krasnosel'skii, MA: Topological Methods in the Theory of Nonlinear Integral Equations. Translated by A. H. Armstrong; translation edited by J. Burlak. A Pergamon Press Book. Macmillan Co., New York (1964)

17. Rabinowitz, PH: On bifurcation from infinity. J. Differ. Equ. 14, 462-475 (1973)

18. Rabinowitz, PH: Some global results for nonlinear eigenvalue problems. J. Funct. Anal. 7, 487-513 (1971)

19. Dancer, EN: On the structure of solutions of non-linear eigenvalue problems. Indiana Univ. Math. J. 23, 1069-1076 (1973/1974)

20. Dancer, EN: Bifurcation from simple eigenvalues and eigenvalues of geometric multiplicity one. Bull. Lond. Math. Soc 34(5), 533-538 (2002)

21. López-Gómez, J: Spectral Theory and Nonlinear Functional Analysis. Chapman \& Hall/CRC Research Notes in Mathematics, vol. 426. Chapman \& Hall/CRC, Boca Raton (2001)

22. Birindelli, I, Mitidieri, Ė, Svirs, G: Existence of the principal eigenvalue for cooperative elliptic systems in a general domain. Differ. Uravn. 35(3), 325-333, 429 (1999)

23. Costa, DG, Magalhães, CA: A variational approach to subquadratic perturbations of elliptic systems. J. Differ. Equ. 111(1), 103-122 (1994)

24. Girg, P, Takáč, P: Bifurcations of positive and negative continua in quasilinear elliptic eigenvalue problems. Ann. Henri Poincaré 9(2), 275-327 (2008) 